\title{
Interaction of waves with a body floating on polynya between two semi-infinite ice sheets
}

Z.F. Li ${ }^{\text {a }}$, Y.Y. Shi ${ }^{\text {b }}$, and G.X. Wu ${ }^{\text {a,c,* }}$

a School of Naval Architecture and Ocean Engineering, Jiangsu University of Science and Technology, Zhenjiang 212003, China

${ }^{\mathrm{b}}$ College of Shipbuilding Engineering, Harbin Engineering University, Harbin 150001, China

${ }^{c}$ Department of Mechanical Engineering, University College London, Torrington Place, London WC1E 7JE, UK

\begin{abstract}
The interaction of waves with a two-dimensional body floating on polynya between two semi-infinite ice sheets is investigated, based on a hybrid method utilizing a simple source function and eigenfunction matching. The ice sheet is modelled as a continuous thin elastic plate with uniform properties, while the fluid flow is described by the velocity potential. In the polynya, an integral equation is established by using the simple source function. In the two exterior ice covered regions, the potential is expanded in terms of eigenfunctions which satisfy the governing equation and all boundary conditions apart from that on the interface with the inner region. The unknown coefficients in the expansion and the boundary integral equation in the inner region are solved together by enforcing the continuity conditions of the pressure and normal velocity on the interface. The effectiveness and accuracy of the hybrid method is demonstrated through comparison with published results for a submerged cylinder and a floating rectangular body. Simulations are then carried out for a floating elliptical cylinder. Extensive results for the hydrodynamic force and motion response are provided, and the effects of ice draught as well as the body shape are investigated.
\end{abstract}

Keywords: ice sheet; floating body; polynya; simple source function; eigenfunction expansion; hybrid method

\section{Introduction}

The latest developments in Arctic engineering motivated by possible new routes for shipping and sources for resource extraction have led to an increased interest in wave/body/ice interactions. A typical case is that a ship navigates across the ice covered region, in a channel opened by an

\footnotetext{
* Corresponding author. Permanent address: Department of Mechanical Engineering, University College London, Torrington Place, London WCIE 7JE, UK. Tel.: +44 207679 3870; fax: +44 2073880180.

E-mail address: g.wu@ucl.ac.uk (G.X.Wu)
} 
icebreaker (Appolonov et al., 2013). In such a case, the ship is floating on a strip of water surface confined between two semi-infinite ice sheets. Due to the wave reflection and transmission when propagating across the ice covered region (Fox and Squire, 1990), highly complex behaviour of the ship motion can be expected. Thus, an efficient numerical tool is adopted to investigate the characteristics of the motion.

There has been strong interest in wave/ice sheet interaction problems. Much of the early work was concerned with marginal sea, as reviewed by Squire et al. (1995), and more recently Squire (2007) and Squire (2011). Fox and Squire (1990) considered the reflection and transmission of free surface waves impinging the ice sheet in the normal direction based on the eigenfunction method. This was extended to the case of an oblique incident wave by Fox and Squire (1994). Sahoo et al. (2001) solved similar problems through defining an inner product with orthogonality in the eigenfunction expansion method, and the influence of edge conditions was analyzed. Linton and Chung (2003) used the residue calculus technique and solved the problem in which the oblique incident wave was from the open sea into the ice covered region or the other way round. Porter and Evans (2007) solved the problem of a water surface covered by an elastic sheet with parallel finite straight cracks. Meylan and Sturova (2009) considered the transient problem of a finite elastic plate on the free surface. Williams and Porter (2009) studied the problem of wave propagation through the ice sheets with non uniform draught. Mohapatra et al. (2013) analyzed the effects of compressive force on the wave/elastic sheet interactions. The Wiener-Hopf method was also widely used to solve this type of problem. Typical work includes those by Evans and Davies (1968), Balmforth and Craster (1999), Tkacheva (2001), Chung and Fox (2002) and Tkacheva (2004).

There has also been increasing interest in wave/body/ice interaction. Sturova (2015) used the boundary element method together with Green functions for a submerged body. Ren et al. (2016) used a semi analytical solution for a rectangular body floating on the water surface. The obtained explicit equations provided some insight into this kind of problem, but the method was limited to this particular body shape only.

The velocity potential problem related to body/free surface interaction is usually solved by the boundary element method. It converts the governing Laplace equation in the fluid domain into an integral equation over its boundary together with the Green function. When the free surface motion and the body motion are small, linearization of the boundary condition can be applied and 
it can be imposed on the mean surface of the fluid domain. As adding a harmonic function which satisfies the governing Laplace equation into the Green function will not change its nature, the Green function adopted in the boundary integral equation can be chosen in such a way that it satisfies all the boundary conditions apart from that on the body surface (Wehausen and Laitone, 1960), which is commonly called the free surface Green function. As a result, the boundary integral equation needs to be applied only to the body surface (Newman, 1977). This can significantly reduce the effort required in surface discretization and surface integration. However, the Green function is in an integral form, which, in the three dimensional problem, can be either a double integral or has an integrand involving complex special functions. The calculation of the Green function itself can be very time consuming. This led to the development of the hybrid method.

Yeung (1975) and Yeung and Bouger (1979) developed a hybrid method in which the velocity potential away from the body was expanded into an orthogonal series of eigenfunctions. Near the body, the simple source function $\ln (r)$ was used, where $r$ is the distance between the source point and the field point. This avoided extensive discretization while it retained the advantage of the boundary element method. Related methods adopted a localized finite element method while the boundary integral was performed on the outer surface of the finite element mesh. Although the free surface Green function was still used, the surface integration was performed on a much simpler rectangular shape. Typical work includes those by Bai and Yeung (1974), Eatock Taylor and Zietsman (1981) for wave radiation and diffraction without forward speed, and by Wu and Eatock Taylor (1987) and Wu (1991) for problems with forward speed. A near field boundary element method coupled with the finite element method in the far field was also used for the nonlinear wave/body interaction problems (Wu and Eatock Taylor, 2003).

With the developments in computer power and numerical algorithms for the free surface Green function, it is more common nowadays to adopt the boundary element method directly on the body surface for the linear radiation and diffraction problems. However, the latest developments in Arctic engineering have led to renewed interest in the hybrid method. In such a case, the water surface is no longer entirely 'free' everywhere and part of it is covered by the ice sheet. The Green function which satisfies both boundary conditions on the free surface and ice sheet is less straightforward to obtain than the free surface only problem (Wehausen and Laitone, 1960) or the ice cover only problem (Sturova, 2013). Usually it is obtained from the matched eigenfunction method. Different series are used in each sub-domain. The unknown coefficients in each series are 
found by imposing a continuity condition at the interface. Sturova (2015) solved the problem for the Green function with two ice sheets of zero draught. This was then used for a submerged body in polynya and only the body surface discretization was needed. Unlike the free surface only problem, the Green function would have to be found for a different polynya or a different ice sheet draught.

Therefore, the hybrid method will be reintroduced in this work, and be applied to the interaction of waves with a horizontal cylinder of arbitrary shape floating on polynya. The total fluid domain is divided into three sub-regions, i.e. one interior region with the free surface, two exterior regions with the ice sheet. In the interior region, the simple source function is used to construct the boundary integral equation over its boundary. In the two exterior regions, the potential is expanded in terms of the eigenfunctions which satisfy all the boundary conditions apart from that on the interface with the interior region. The unknown coefficients in the expansion and the integral equation are solved together through enforcing continuity of pressure and normal velocity on the interface. The effectiveness and accuracy of the proposed numerical scheme is demonstrated through two typical cases, i.e. a submerged cylinder and a floating rectangular body. Comparison is made with the published results and very good agreement is achieved. Simulations are then carried out for a floating elliptical cylinder. Extensive results are provided through the hydrodynamic force and motion response, and the effects of ice draught as well as the body shape are investigated.

\section{Mathematical model and numerical procedures}

\subsection{Mathematical model}

The problem of wave interaction with a body floating on an arbitrary position of polynya between two semi-infinite ice sheets is sketched in Fig. 1. The width of the body at the water surface and its draught are $a$ and $b$ respectively. A Cartesian coordinate system $O-x z$ is defined, with the $x$-axis along the undisturbed mean free surface, and the $z$-axis pointing vertically upwards. The oscillation of the body is excited by an incident wave propagating underneath the left ice sheet along the $x$-axis. When the body is at its equilibrium position, the $z$-axis passes through the centre of mass of the body. The fluid with density $\rho$ and constant depth $H$ is assumed to be inviscid, incompressible and homogeneous, and its motion to be irrotational. Thus the velocity potential $\Phi$ can be introduced to describe the fluid flow. The ice sheet, which begins from $x_{j}$ and extends to infinity on each side, is modelled as a continuous elastic plate with uniform properties, i.e. thickness $h_{j}$, draught $d_{j}$, density $\rho_{j}$, Young's modulus $E_{j}$, Poisson's ration $v_{j}$. Here the 
subscripts $j=1,2$ denote the left and right hand side ice sheets respectively. The justification of such a model for the ice sheet has been discussed by Robin (1963) and Squire et al. (1988) based on the field measurements.

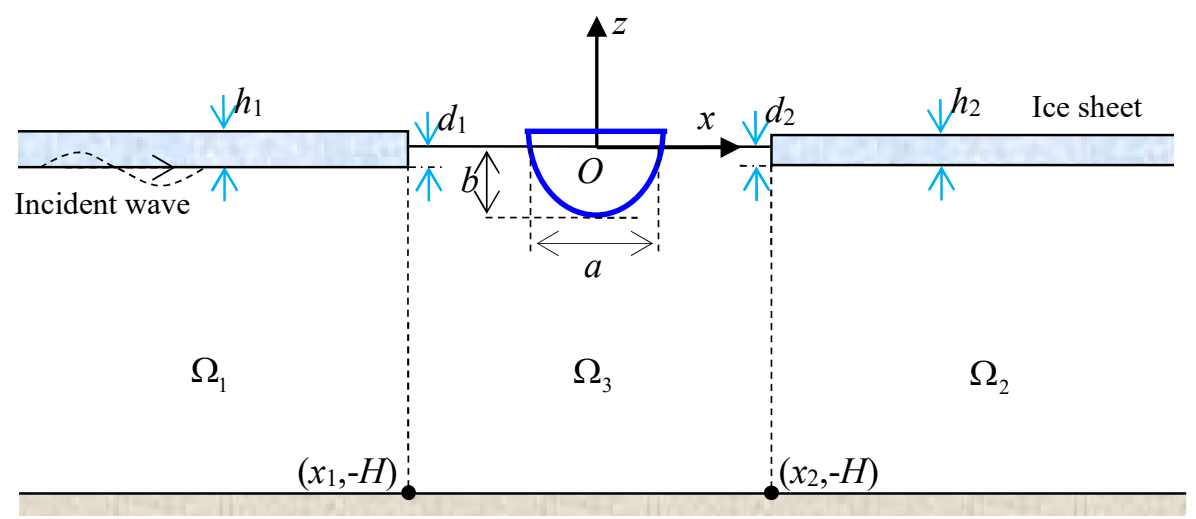

Fig. 1. Sketch of the problem.

Under the assumption that the amplitude of wave motion is small compared to its length and the dimension of the body, the linearized velocity potential theory can be used. When the motion is sinusoidal in time with frequency $\omega$, the total potential can be written as (Wehausen and Laitone, 1960)

$$
\Phi(x, z, t)=\operatorname{Re}\left[\alpha_{0} \phi_{0}(x, z) \mathrm{e}^{\mathrm{i} \omega t}\right]+\operatorname{Re}\left[\sum_{i=1}^{3} \mathrm{i} \omega \alpha_{i} \phi_{i}(x, z) \mathrm{e}^{\mathrm{i} \omega t}\right]
$$

where $\phi_{0}$ contains the incident potential $\phi_{I}$ and diffracted potential $\phi_{D}, \alpha_{0}$ is the amplitude of the incident wave; $\phi_{i}(i=1,2,3)$ is the radiation potential due to body oscillation with complex amplitude $\alpha_{i}$ in three degrees of freedom: translations in $x$ and $z$ directions respectively and rotation about $y$ pointing into the paper. Mass conservation requires that the potential $\phi_{i}$ satisfies Laplace's equation

$$
\nabla^{2} \phi_{i}=0,(i=0,1,2,3)
$$

throughout the fluid. The combination of the linearized dynamic and kinematic free surface boundary conditions gives

$$
-\omega^{2} \phi_{i}+g \frac{\partial \phi_{i}}{\partial z}=0,\left(x_{1}<x<x_{2}, \quad z=0\right)
$$

where $g$ is the acceleration due to gravity. On the ice sheets, the boundary condition can be written as (Squire et al., 1995)

$$
\left(L_{j} \frac{\partial^{4}}{\partial x^{4}}-m_{j} \omega^{2}+\rho g\right) \frac{\partial \phi_{i}}{\partial z}-\rho \omega^{2} \phi_{i}=0,\left(|x| \geq\left|x_{j}\right|, \quad z=-d_{j}, \quad j=1,2\right)
$$

where $L_{j}=E h_{j}^{3} /\left[12\left(1-v_{j}^{2}\right)\right]$ is the effective flexural rigidity of the ice sheet, and $m_{j}=h_{j} \rho_{j}$ is its mass per unit area. On the vertical surface of the ice sheet, the impermeable condition yields

$$
\frac{\partial \phi_{i}}{\partial x}=0,\left(x=x_{j},-d_{j} \leq z \leq 0\right)
$$


The impermeable condition on the body surface is

$$
\frac{\partial \phi_{0}}{\partial n}=0 \text { and } \frac{\partial \phi_{i}}{\partial n}=n_{i},(i=1,2,3)
$$

where $n_{1}$ and $n_{2}$ are the $x, z$ components of the unit normal vector $\vec{n}$ pointing into the body, $n_{3}=\left(z-z^{\prime}\right) n_{1}-\left(x-x^{\prime}\right) n_{2}$ is the component related to the rotational mode, with $\left(x^{\prime}, z^{\prime}\right)$ as the rotational centre. The boundary condition on the flat seabed can be written as

$$
\frac{\partial \phi_{i}}{\partial z}=0,(-\infty<x<+\infty, z=-H)
$$

The radiation condition far away requires the wave to propagate outwards

$$
\begin{gathered}
\lim _{x \rightarrow-\infty}\left[\frac{\partial\left(\phi_{i}-\delta_{0, i} \phi_{I}\right)}{\partial x}-\kappa_{0}^{(1)}\left(\phi_{i}-\delta_{0, i} \phi_{I}\right)\right]=0 \\
\lim _{x \rightarrow+\infty}\left(\frac{\partial \phi_{i}}{\partial x}+\kappa_{0}^{(2)} \phi_{i}\right)=0
\end{gathered}
$$

Here the Kronecker delta function $\delta_{p, q}=1$ if $p=q$ and $\delta_{p, q}=0$ if $p \neq q$. Thus $\phi_{I}$ is included only when $i=0$ and it does not affect wave radiation. $\kappa_{0}^{(1)}$ and $\kappa_{0}^{(2)}$ are the purely positive imaginary roots of the corresponding dispersion equations in regions $\Omega_{1}$ and $\Omega_{2}$ respectively, which will be discussed in detail below.

\subsection{Numerical procedure}

\subsubsection{Eigenfunction expansion in regions 1 and 2}

In the ice-covered regions $\Omega_{1}$ and $\Omega_{2}$, the velocity potential is written in terms of the corresponding eigenfunctions. Each base function satisfies the Laplace equation, upper surface and bottom boundary conditions, as well as the far field radiation conditions. We have

$$
\begin{gathered}
\phi_{i}^{(1)}=\delta_{0, i} \phi_{I}+\sum_{m=-2}^{\infty} R_{i, m} \psi_{m}^{(1)} \\
\phi_{i}^{(2)}=\sum_{m=-2}^{\infty} T_{i, m} \psi_{m}^{(2)}
\end{gathered}
$$

where

$$
\begin{gathered}
\phi_{I}=\frac{g}{\mathrm{i} \omega} \mathrm{e}^{-\kappa_{0}^{(1)}\left(x-x_{1}\right)} \frac{\cos \left[\kappa_{0}^{(1)}(H+z)\right]}{\cos \left[\kappa_{0}^{(1)}\left(H-d_{1}\right)\right]} \\
\psi_{m}^{(j)}=\mathrm{e}^{\kappa_{m}^{(j)}\left(x_{j}-x\right) \operatorname{sgn}\left(x-x_{j}\right)} \frac{\cos \left[\kappa_{m}^{(j)}(H+z)\right]}{\cos \left[\kappa_{m}^{(j)}\left(H-d_{j}\right)\right]}
\end{gathered}
$$

with $\operatorname{sgn}\left(x-x_{j}\right)=1$ if $x-x_{j}>0$ and $\operatorname{sgn}\left(x-x_{j}\right)=-1$ if $x-x_{j}<0$. It should be noted that $R_{i, m}, T_{i, m}$ are the unknown coefficients to be determined, and $\kappa_{m}^{(j)}$ is the root of the following dispersion equations

$$
-\kappa_{m}^{(j)} \tan \left[\kappa_{m}^{(j)}\left(H-d_{j}\right)\right]=\frac{\rho \omega^{2}}{L_{j}\left(\kappa_{m}^{(j)}\right)^{4}+\rho g-m_{j} \omega^{2}}
$$

which can be obtained by substituting Eqs. (10) and (11) respectively into Eq. (4). It should be 
pointed out that $\kappa_{0}^{(j)}$ is the purely positive imaginary root, $\kappa_{-2}^{(j)}$ and $\kappa_{-1}^{(j)}$ are a pair of conjugated complex roots with positive real parts, $\kappa_{m}^{(j)}(m=1,2, \ldots)$ are purely positive real roots.

Following Ren et al. (2016) and using Green's second theorem over the boundary $S_{i}$ of $\Omega_{i}$, we have

$$
\oint_{S_{1}}\left[\left(\phi_{i}^{(1)}-\delta_{0, i} \phi_{I}\right) \frac{\partial \psi_{m}^{(1)}}{\partial n}-\frac{\partial\left(\phi_{i}^{(1)}-\delta_{0, i} \phi_{I}\right)}{\partial n} \psi_{m}^{(1)}\right] \mathrm{d} S=0 \text { in } \Omega_{1}
$$

and

$$
\oint_{S_{2}}\left(\phi_{i}^{(2)} \frac{\partial \psi_{m}^{(2)}}{\partial n}-\frac{\partial \phi_{i}^{(2)}}{\partial n} \psi_{m}^{(2)}\right) \mathrm{d} S=0 \text { in } \Omega_{2}
$$

We notice that the functions $\left(\phi_{i}^{(1)}-\delta_{0, i} \phi_{I}\right)$ and $\psi_{m}^{(1)}, \phi_{i}^{(2)}$ and $\psi_{m}^{(2)}$ satisfy the same bottom, radiation and ice sheet surface conditions. As a result, only the ice sheet surface and interface will be retained in the integration. Using integration by parts for the ice sheet surface, as in Ren et al. (2016), we can rewrite Eqs. (15) and (16) as

$$
\begin{aligned}
& \int_{-H}^{-d_{1}}\left(\phi_{i}^{(1)} \frac{\partial \psi_{m}^{(1)}}{\partial x}-\frac{\partial \phi_{i}^{(1)}}{\partial x} \psi_{m}^{(1)}\right) \mathrm{d} z \\
& \quad+\frac{L_{1}}{\rho \omega^{2}}\left(\frac{\partial^{4} \phi_{i}^{(1)}}{\partial x^{3} \partial z} \frac{\partial \psi_{m}^{(1)}}{\partial z}-\frac{\partial^{3} \phi_{i}^{(1)}}{\partial x^{2} \partial z} \frac{\partial^{2} \psi_{m}^{(1)}}{\partial z \partial x}+\frac{\partial^{3} \psi_{m}^{(1)}}{\partial x^{2} \partial z} \frac{\partial^{2} \phi_{i}^{(1)}}{\partial z \partial x}-\frac{\partial^{4} \psi_{m}^{(1)}}{\partial x^{3} \partial z} \frac{\partial \phi_{i}^{(1)}}{\partial z}\right)_{z=-d_{1}} \\
& =\delta_{0, i} \int_{-H}^{-d_{1}}\left(\phi_{I} \frac{\partial \psi_{m}^{(1)}}{\partial x}-\frac{\partial \phi_{I}}{\partial x} \psi_{m}^{(1)}\right) \mathrm{d} z \\
& \quad+\frac{\delta_{0, i} L_{1}}{\rho \omega^{2}}\left(\frac{\partial^{4} \phi_{I}}{\partial x^{3} \partial z} \frac{\partial \psi_{m}^{(1)}}{\partial z}-\frac{\partial^{3} \phi_{I}}{\partial x^{2} \partial z} \frac{\partial^{2} \psi_{m}^{(1)}}{\partial z \partial x}+\frac{\partial^{3} \psi_{m}^{(1)}}{\partial x^{2} \partial z} \frac{\partial^{2} \phi_{I}}{\partial z \partial x}-\frac{\partial^{4} \psi_{m}^{(1)}}{\partial x^{3} \partial z} \frac{\partial \phi_{I}}{\partial z}\right)_{z=-d_{1}}
\end{aligned}
$$

and

$$
\begin{aligned}
& \int_{-H}^{-d_{2}}\left(\phi_{i}^{(2)} \frac{\partial \psi_{m}^{(2)}}{\partial x}-\frac{\partial \phi_{i}^{(2)}}{\partial x} \psi_{m}^{(2)}\right) \mathrm{d} z \\
& \quad+\frac{L_{2}}{\rho \omega^{2}}\left(\frac{\partial^{4} \phi_{i}^{(2)}}{\partial x^{3} \partial z} \frac{\partial \psi_{m}^{(2)}}{\partial z}-\frac{\partial^{3} \phi_{i}^{(2)}}{\partial x^{2} \partial z} \frac{\partial^{2} \psi_{m}^{(2)}}{\partial z \partial x}+\frac{\partial^{3} \psi_{m}^{(2)}}{\partial x^{2} \partial z} \frac{\partial^{2} \phi_{i}^{(2)}}{\partial z \partial x}-\frac{\partial^{4} \psi_{m}^{(2)}}{\partial x^{3} \partial z} \frac{\partial \phi_{i}^{(2)}}{\partial z}\right)_{z=-d_{2}} \text { at } x=x_{2} \\
& =0
\end{aligned}
$$

The last terms on the left hand side of Eqs. (17) and (18) will depend on the condition at the ice sheet edge. Without loss of generality, we may assume a free edge which gives

$$
\frac{\partial^{2}}{\partial x^{2}}\left(\frac{\partial \phi_{i}}{\partial z}\right)=\frac{\partial^{3}}{\partial x^{3}}\left(\frac{\partial \phi_{i}}{\partial z}\right)=0,\left(x=x_{j}, \quad z=-d_{j}\right)
$$

Eqs. (17) and (18) can then be simplified as

$$
\int_{-H}^{-d_{1}}\left(\phi_{i}^{(1)} \frac{\partial \psi_{m}^{(1)}}{\partial x}-\frac{\partial \phi_{i}^{(1)}}{\partial x} \psi_{m}^{(1)}\right) \mathrm{d} z+\frac{L_{1}}{\rho \omega^{2}}\left(\frac{\partial^{3} \psi_{m}^{(1)}}{\partial x^{2} \partial z} \frac{\partial^{2} \phi_{i}^{(1)}}{\partial z \partial x}-\frac{\partial^{4} \psi_{m}^{(1)}}{\partial x^{3} \partial z} \frac{\partial \phi_{i}^{(1)}}{\partial z}\right)_{z=-d_{1}}=\delta_{0, i} I_{i} \quad \text { at } \quad x=x_{1}
$$

and

$$
\int_{-H}^{-d_{2}}\left(\phi_{i}^{(2)} \frac{\partial \psi_{m}^{(2)}}{\partial x}-\frac{\partial \phi_{i}^{(2)}}{\partial x} \psi_{m}^{(2)}\right) \mathrm{d} z+\frac{L_{2}}{\rho \omega^{2}}\left(\frac{\partial^{3} \psi_{m}^{(2)}}{\partial x^{2} \partial z} \frac{\partial^{2} \phi_{i}^{(2)}}{\partial z \partial x}-\frac{\partial^{4} \psi_{m}^{(2)}}{\partial x^{3} \partial z} \frac{\partial \phi_{i}^{(2)}}{\partial z}\right)_{z=-d_{2}}=0 \text { at } x=x_{2}
$$

where 


$$
\begin{aligned}
I_{i}= & \int_{-H}^{-d_{1}}\left(\phi_{I} \frac{\partial \psi_{m}^{(1)}}{\partial x}-\frac{\partial \phi_{I}}{\partial x} \psi_{m}^{(1)}\right) \mathrm{d} z \\
& +\frac{L_{1}}{\rho \omega^{2}}\left(\frac{\partial^{4} \phi_{I}}{\partial x^{3} \partial z} \frac{\partial \psi_{m}^{(1)}}{\partial z}-\frac{\partial^{3} \phi_{I}}{\partial x^{2} \partial z} \frac{\partial^{2} \psi_{m}^{(1)}}{\partial z \partial x}+\frac{\partial^{3} \psi_{m}^{(1)}}{\partial x^{2} \partial z} \frac{\partial^{2} \phi_{I}}{\partial z \partial x}-\frac{\partial^{4} \psi_{m}^{(1)}}{\partial x^{3} \partial z} \frac{\partial \phi_{I}}{\partial z}\right)_{z=-d_{1}}
\end{aligned}
$$

is known. The first term at the interface in Eq. (20) or (21) will then be matched below with the solution from the region $\Omega_{3}$.

\subsubsection{Boundary integral equation in region 3}

In region $\Omega_{3}$, which is bounded by the body surface $S_{0}$, free surface $S_{F}$, two vertical interfaces $\Sigma_{1}$ and $\Sigma_{2}$, and seabed $S_{B}$, the problem in the fluid domain can be converted into an integral equation over its boundary $S$ using the simple source function. Through Green's identity, we have

$$
\alpha(p) \phi_{i}^{(3)}(p)=\int_{S}\left[G(p, q) \frac{\partial \phi_{i}^{(3)}(q)}{\partial n_{q}}-\frac{\partial G(p, q)}{\partial n_{q}} \phi_{i}^{(3)}(q)\right] \mathrm{d} S_{q}
$$

where $S=S_{0}+S_{F}+\Sigma_{1}+\Sigma_{2}+S_{B}, \alpha(p)$ is the solid angle at the field point $p(x, z)$, while $q(\xi, \zeta)$ is the source point. The simple source function in Eq. (23) is defined as

$$
G(p, q)=\ln \left(1 / r_{1}\right)+\ln \left(1 / r_{2}\right)
$$

where $r_{1}=\sqrt{(x-\xi)^{2}+(z-\zeta)^{2}}$ and $r_{2}=\sqrt{(x-\xi)^{2}+(z+\zeta+2 H)^{2}}$ respectively are the distance between the field and the source point, and the distance between the field point and the mirror of the source point with respect to the seabed. As $\partial G / \partial z=0$ on $z=-H$, the integral over the flat seabed in Eq. (23) can be omitted (Bai and Yeung, 1974).

Invoking the boundary condition on the vertical surface of each ice sheet edge in Eq. (5), we can write Eq. (23) as

$$
\begin{aligned}
\alpha(p) \phi_{i}^{(3)}(p) & =\int_{S_{0}+S_{F}}\left[G(p, q) \frac{\partial \phi_{i}^{(3)}(q)}{\partial n_{q}}-\frac{\partial G(p, q)}{\partial n_{q}} \phi_{i}^{(3)}(q)\right] \mathrm{d} S_{q} \\
& -\int_{-H}^{0}\left[\frac{\partial G(p, q)}{\partial n_{q}} \phi_{i}^{(3)}(q)\right]_{\xi=x_{1}} \mathrm{~d} \zeta-\int_{-H}^{0}\left[\frac{\partial G(p, q)}{\partial n_{q}} \phi_{i}^{(3)}(q)\right]_{\xi=x_{2}} \mathrm{~d} \zeta \\
& +\int_{-H}^{-d_{1}}\left[G(p, q) \frac{\partial \phi_{i}^{(3)}(q)}{\partial n_{q}}\right]_{\xi=x_{1}} \mathrm{~d} \zeta+\int_{-H}^{-d_{2}}\left[G(p, q) \frac{\partial \phi_{i}^{(3)}(q)}{\partial n_{q}}\right]_{\xi=x_{2}} \mathrm{~d} \zeta
\end{aligned}
$$

On the vertical interface at $x=x_{j}$, we may expand the velocity potential $\phi_{i}^{(3)}$ in the following series form

$$
\phi_{i}^{(3)}\left(x_{j}, z\right)=\sum_{m=0}^{\infty} C_{i, m}^{(j)} \psi_{m}^{(3)}
$$

where

$$
\psi_{m}^{(3)}=\frac{\cos \left[\kappa_{m}^{(3)}(H+z)\right]}{\cos \left(\kappa_{m}^{(3)} H\right)}
$$

with $\kappa_{m}^{(3)}$ being the root of the following dispersion equation 


$$
-\kappa_{m}^{(3)} \tan \left(\kappa_{m}^{(3)} H\right)=\frac{\omega^{2}}{g}
$$

which is obtained by substituting Eq. (26) into Eq. (3). We notice that $\kappa_{0}^{(3)}$ is the purely positive imaginary root, $\kappa_{m}^{(3)}(m=1,2, \ldots)$ are the purely positive real roots. Substituting Eq. (26) into Eq. (25), we have

$$
\begin{aligned}
\alpha(p) \phi_{i}^{(3)}(p) & =\int_{S_{0}}\left[G(p, q) \frac{\partial \phi_{i}^{(3)}(q)}{\partial n_{q}}-\frac{\partial G(p, q)}{\partial n_{q}} \phi_{i}^{(3)}(q)\right] \mathrm{d} S_{q} \\
& +\int_{S_{F}}\left[G(p, q) \frac{\partial \phi_{i}^{(3)}(q)}{\partial \zeta}-\frac{\partial G(p, q)}{\partial \zeta} \phi_{i}^{(3)}(q)\right] \mathrm{d} S_{q} \\
& +\sum_{m=0}^{\infty} C_{i, m}^{(1)} \int_{-H}^{0}\left[\frac{\partial G(p, q)}{\partial \xi} \psi_{m}^{(3)}(\zeta)\right]_{\xi=x_{1}} \mathrm{~d} \zeta \\
& -\sum_{m=0}^{\infty} C_{i, m}^{(2)} \int_{-H}^{0}\left[\frac{\partial G(p, q)}{\partial \xi} \psi_{m}^{(3)}(\zeta)\right]_{\xi=x_{2}} \mathrm{~d} \zeta \\
& -\int_{-H}^{-d_{1}}\left[G(p, q) \frac{\partial \phi_{i}^{(3)}(q)}{\partial \xi}\right]_{\xi=x_{1}} \mathrm{~d} \zeta+\int_{-H}^{-d_{2}}\left[G(p, q) \frac{\partial \phi_{i}^{(3)}(q)}{\partial \xi}\right]_{\xi=x_{2}} \mathrm{~d} \zeta
\end{aligned}
$$

\subsubsection{Matching on the interfaces}

The matching procedure is to enforce the continuity conditions of the pressure and the normal velocity on the vertical interfaces, namely

$$
\phi_{i}^{(j)}=\phi_{i}^{(3)}, \frac{\partial \phi_{i}^{(j)}}{\partial x}=\frac{\partial \phi_{i}^{(3)}}{\partial x} \quad\left(x=x_{j}, j=1,2\right)
$$

To achieve this, the normal derivatives in regions $\Omega_{1}$ and $\Omega_{2}$ obtained from Eqs. (10) and (11) are substituted into the integral in Eq. (29) along the interface. Together with Eq. (3), this leads to

$$
\begin{aligned}
\alpha(p) \phi_{i}^{(3)}(p) & =\int_{S_{0}}\left[G(p, q) \frac{\partial \phi_{i}^{(3)}(q)}{\partial n_{q}}-\frac{\partial G(p, q)}{\partial n_{q}} \phi_{i}^{(3)}(q)\right] \mathrm{d} S_{q} \\
& +\int_{S_{F}}\left[G(p, q) \frac{\omega^{2}}{g} \phi_{i}^{(3)}(q)-\frac{\partial G(p, q)}{\partial \zeta} \phi_{i}^{(3)}(q)\right] \mathrm{d} S_{q} \\
& +\sum_{m=0}^{\infty} C_{i, m}^{(1)} F_{m}\left(x, z, x_{1}\right)-\sum_{m=0}^{\infty} C_{i, m}^{(2)} F_{m}\left(x, z, x_{2}\right) \\
& +\delta_{0, i} \frac{g}{i} G_{0}^{(1)}\left(x, z, x_{1}\right)-\sum_{m=-2}^{\infty} R_{i, m} G_{m}^{(1)}\left(x, z, x_{1}\right) \\
& +\sum_{m=-2}^{\infty} T_{i, m} G_{m}^{(2)}\left(x, z, x_{2}\right)
\end{aligned}
$$

where

$$
\begin{aligned}
F_{m}(x, z, \xi) & =\int_{-H}^{0} \frac{\partial G(p, q)}{\partial \xi} \psi_{m}^{(3)}(\zeta) \mathrm{d} \zeta \\
G_{m}^{(j)}(x, z, \xi) & =\int_{-H}^{-d_{j}} G(p, q) \frac{\partial \psi_{m}^{(j)}(q)}{\partial \xi} \mathrm{d} \zeta
\end{aligned}
$$

The integration along the depth in the above equations can be performed either numerically or analytically based on the exponential integral (Yeung and Bouger, 1979). When $p$ is located at the interface $x=x_{j}$, the term on the left hand side of Eq. (31) may be replaced by the expansion 
in Eq. (26). Multiplying both sides of the equation by $\psi_{m^{\prime}}^{(3)}$, and then integrating the product with respect to $z$ over $z \in[-H, 0]$, we have

$$
\begin{aligned}
& \frac{\pi C_{i, m^{\prime}}^{(j)}}{\cos ^{2}\left(\kappa_{m^{\prime}}^{(3)} H\right)}\left[\frac{H}{2}+\frac{\sin \left(2 \kappa_{m^{\prime}}^{(3)} H\right)}{4 \kappa_{m^{\prime}}^{(3)}}\right] \\
& \quad=\int_{-H}^{0} \psi_{m^{\prime}}^{(3)}(z) \mathrm{d} z \int_{S_{0}}\left[G(p, q) \frac{\partial \phi_{i}^{(3)}(q)}{\partial n_{q}}-\frac{\partial G(p, q)}{\partial n_{q}} \phi_{i}^{(3)}(q)\right] \mathrm{d} S_{q} \\
& \quad+\int_{-H}^{0} \psi_{m^{\prime}}^{(3)}(z) \mathrm{d} z \int_{S_{F}}\left[G(p, q) \frac{\omega^{2}}{g} \phi_{i}^{(3)}(q)-\frac{\partial G(p, q)}{\partial \zeta} \phi_{i}^{(3)}(q)\right] \mathrm{d} S_{q} \\
& \quad+\sum_{m=0}^{\infty} C_{i, m}^{(1)} F F_{m^{\prime}, m}\left(x_{j}, x_{1}\right)-\sum_{m=0}^{\infty} C_{i, m}^{(2)} F F_{m^{\prime}, m}\left(x_{j}, x_{2}\right) \\
& \quad+\delta_{0, i} \frac{g}{\mathrm{i} \omega} G G_{m^{\prime}, 0}^{(1)}\left(x_{j}, x_{1}\right)-\sum_{m=-2}^{\infty} R_{i, m} G G_{m^{\prime}, m}^{(1)}\left(x_{j}, x_{1}\right) \\
& \quad+\sum_{m=-2}^{\infty} T_{i, m} G G_{m^{\prime}, m}^{(2)}\left(x_{j}, x_{2}\right)
\end{aligned}
$$

where

$$
\begin{aligned}
F F_{m^{\prime}, m}(x, \xi) & =\int_{-H}^{0} \psi_{m^{\prime}}^{(3)}(z) \mathrm{d} z \int_{-H}^{0} \frac{\partial G(p, q)}{\partial \xi} \psi_{m}^{(3)}(\zeta) \mathrm{d} \zeta \\
G G_{m^{\prime}, m}^{(j)}(x, \xi) & =\int_{-H}^{0} \psi_{m^{\prime}}^{(3)}(z) \mathrm{d} z \int_{-H}^{-d_{j}} G(p, q) \frac{\partial \psi_{m}^{(j)}(q)}{\partial \xi} \mathrm{d} \zeta
\end{aligned}
$$

Here we notice that the integration with respect to $\zeta$ can be done in a way similar to that in Eqs. (32) and (33), while the integration with respect to $z$ is done by the standard six point Gauss-Legendre rule (Abramowitz and Stegun, 1965).

The continuity of the potential on the two interfaces is ensured by substituting Eq. (26) into the first term of Eqs. (20) and (21) respectively. This leads to

$$
\int_{-H}^{-d_{1}}\left[\sum_{m^{\prime}=0}^{\infty} C_{i, m^{\prime}}^{(1)} \psi_{m^{\prime}}^{(3)} \frac{\partial \psi_{m}^{(1)}}{\partial x}-\frac{\partial \phi_{i}^{(1)}}{\partial x} \psi_{m}^{(1)}\right] \mathrm{d} z+\frac{L_{1}}{\rho \omega^{2}}\left[\frac{\partial^{3} \psi_{m}^{(1)}}{\partial x^{2} \partial z} \frac{\partial^{2} \phi_{i}^{(1)}}{\partial z \partial x}-\frac{\partial^{4} \psi_{m}^{(1)}}{\partial x^{3} \partial z} \frac{\partial \phi_{i}^{(1)}}{\partial z}\right]_{z=-d_{1}}=\delta_{0, i} I_{i}
$$

at $x=x_{1}$ and

$$
\int_{-H}^{-d_{2}}\left[\sum_{m^{\prime}=0}^{\infty} C_{i, m^{\prime}}^{(2)} \psi_{m^{\prime}}^{(3)} \frac{\partial \psi_{m}^{(2)}}{\partial x}-\frac{\partial \phi_{i}^{(2)}}{\partial x} \psi_{m}^{(2)}\right] \mathrm{d} z+\frac{L_{2}}{\rho \omega^{2}}\left[\frac{\partial^{3} \psi_{m}^{(2)}}{\partial x^{2} \partial z} \frac{\partial^{2} \phi_{i}^{(2)}}{\partial z \partial x}-\frac{\partial^{4} \psi_{m}^{(2)}}{\partial x^{3} \partial z} \frac{\partial \phi_{i}^{(2)}}{\partial z}\right]_{z=-d_{2}}=0
$$

at $x=x_{2}$.

To solve the above problem numerically, the infinite series in Eqs. (10), (11) and (26) are truncated at finite numbers, $M_{1}, M_{2}$ and $M_{3}$ respectively, which can be chosen as $M_{1}=M_{2}=M-2$ and $M_{3}=M$. The surfaces $S_{0}, S_{F}$ in Eq. (29) are divided into $N_{0}$ and $N_{F}$ straight-line segments respectively, on which the velocity potentials are assumed to be constant and the equation is enforced at the middle point of each segment, which means that the solid angle $\alpha(p)$ can always be taken as $\pi$. All these leads to a system of $4 M+4+N_{0}+N_{F}$ linear equations with the same number of unknowns.

\subsection{Hydrodynamic force and body motions}


After solving for the velocity potentials, the pressure can be obtained through the linear Bernoulli equation. Then the hydrodynamic force acting on the body can be obtained by integrating the dynamic pressure over the mean wetted body surface. According to Eq. (1), the hydrodynamic force can be divided into two parts, i.e. the wave exciting force due to the scattering potential, and the added mass and damping coefficient due to the radiation potential. There is also the hydrostatic force due to the variation of the buoyancy during body oscillation. Then the complex motion amplitudes $\alpha_{k}(k=1,2,3)$ can be computed through the following linear equations (Newman, 1977)

$$
\sum_{k=1}^{3}\left[-\omega^{2}\left(m_{j k}+\mu_{j k}\right)+\mathrm{i} \omega \lambda_{j k}+C_{j k}\right] \alpha_{k}=f_{E, j} \alpha_{0}, \quad j=1,2,3
$$

where $k=1,2,3$ represent the sway, heave and roll modes; $m_{j k}$ and $C_{j k}$ are the body mass and hydrostatic restoring coefficients. The terms $\mu_{j k}$ and $\lambda_{j k}$ in Eq. (39) are the added mass and damping coefficient respectively, which can be computed through the following equation

$$
\mu_{j k}-\mathrm{i} \frac{\lambda_{j k}}{\omega}=\rho \int_{S_{0}} \phi_{k} n_{j} \mathrm{~d} S
$$

The term $f_{E, j}$ on the right hand side of Eq. (39) is the exciting force of the wave with unit amplitude and can be given as

$$
f_{E, j}=-\mathrm{i} \omega \rho \int_{S_{0}} \phi_{0}(x, z) n_{j} \mathrm{~d} S
$$

\section{Numerical results and discussions}

In the following numerical results and discussions, we will use dimensionless variables. The basic parameters are chosen as the width of the floating body $a$ at the water surface, density of water $\rho$, and acceleration due to gravity $g$. The other parameters are nondimensionalized via their combinations.

\subsection{Verification of the present hybrid method}

\subsubsection{A submerged cylinder}

We first consider the case for a submerged elliptical cylinder defined as $\left(x-x_{0}\right)^{2} / a^{2}+\left(z-z_{0}\right)^{2} / b^{2}=1$, where $a$ and $b$ are the half axes of the elliptical cylinder in $x$ and $z$ directions, respectively, and $\left(x_{0}, z_{0}\right)$ is the centre of the cylinder, where the rotational centre is located, or $\left(x^{\prime}, z^{\prime}\right)=\left(x_{0}, z_{0}\right)$. The case has been investigated by Sturova (2015) through the Green function method. The Green function satisfies all the boundary conditions apart for that on the body surface. As a result, the boundary integral equation is over the body surface only. However, that Green function is found by the eigenfunction expansion method and it has to be re-derived for every different polynya configuration. 
To demonstrate the accuracy and effectiveness of the procedure, we adopt the same parameters as those in Sturova (2015), i.e. $a=1, b=0.5, \quad\left(x^{\prime}, z^{\prime}\right)=(0,-1), H=25, x_{1}=-x_{2}=-2.5$, $h_{1}=0.025$ and $h_{2}=0.1, m_{1}=0.0225$ and $m_{2}=0.09, L_{1}=0.0356$ and $L_{2}=2.2791$, and $d_{1}=d_{2}=0$. Fig. 2 presents the added mass with two sets of $M, N_{0}$ and $N_{F}$ over a range of frequencies $\sigma=\omega^{2} / g a$, together with the results of Sturova (2015), while Fig. 3 gives the corresponding damping coefficients. We can see that there is no visible difference between the results obtained with $M=70, N_{0}=90, N_{F}=180$ and $M=100, N_{0}=180, N_{F}=360$, which means that convergence has been achieved. It can be also seen that the numerical results are in good agreement with those of Sturova (2015) (note, $\lambda_{13}$ in Sturova (2015) was incorrect and results of Sturova used in Fig. 3 (d) below are provided by her through private communication). The damping coefficients are also computed by the far field formula (see Eq. (B2) of Ren et al. (2016)) and the results are in an excellent agreement with those from the near field formula in Eq. (40). This shows that the hybrid method presented in this paper is effective and accurate.

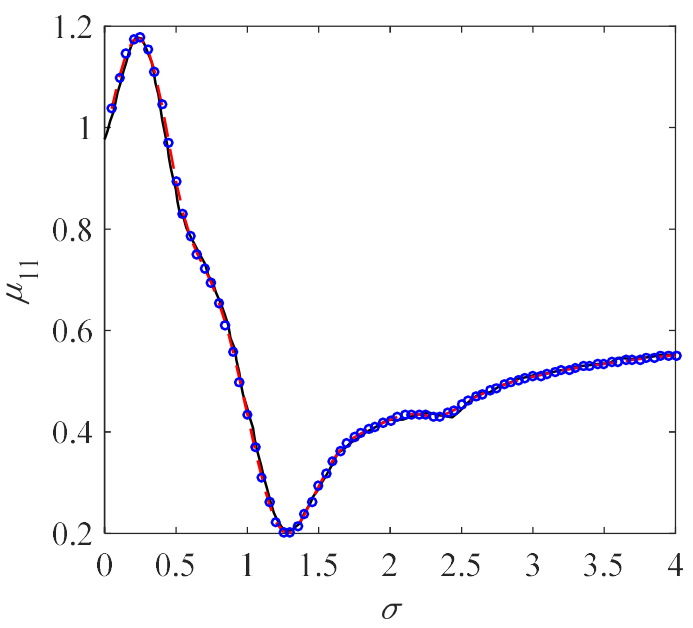

(a)

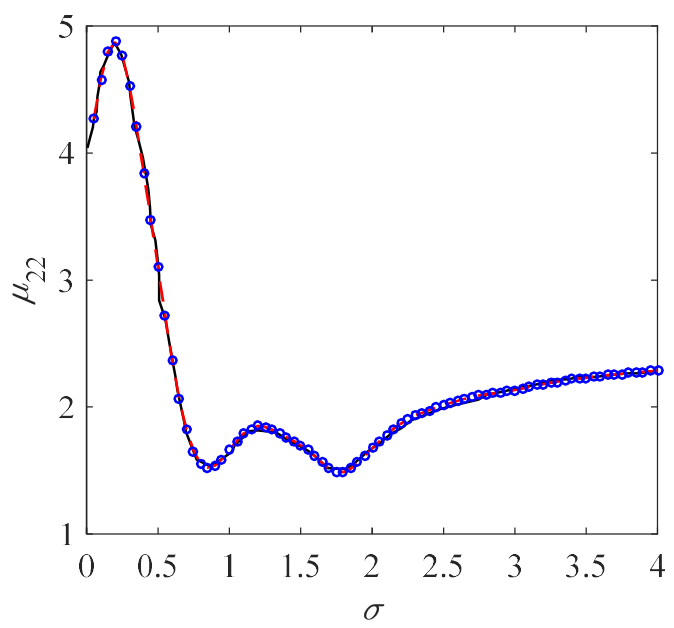

(c)

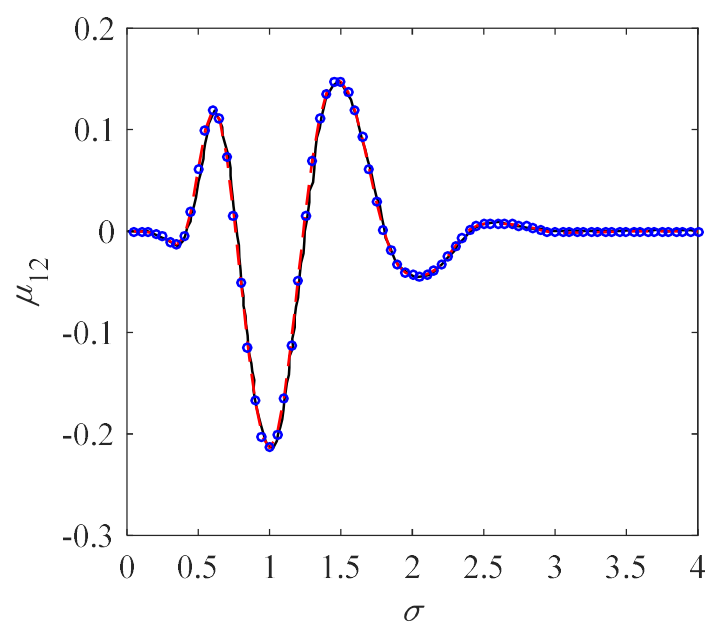

(b)

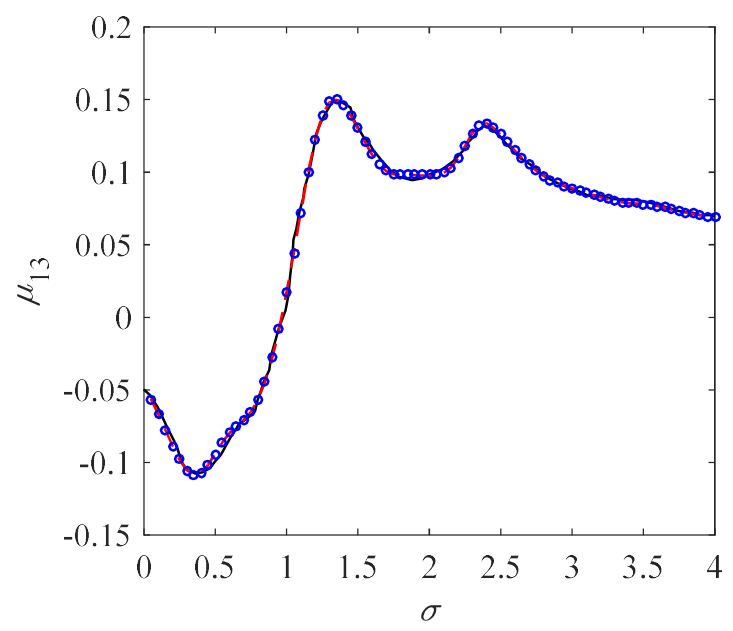

(d) 


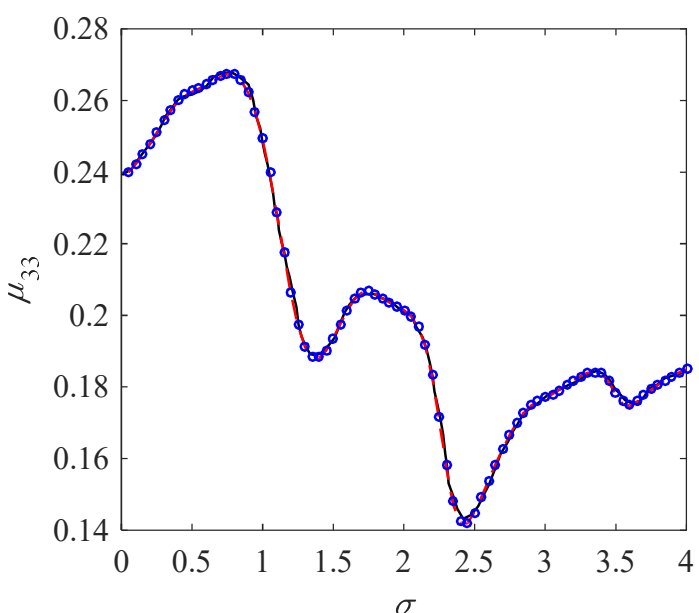

$(e)$

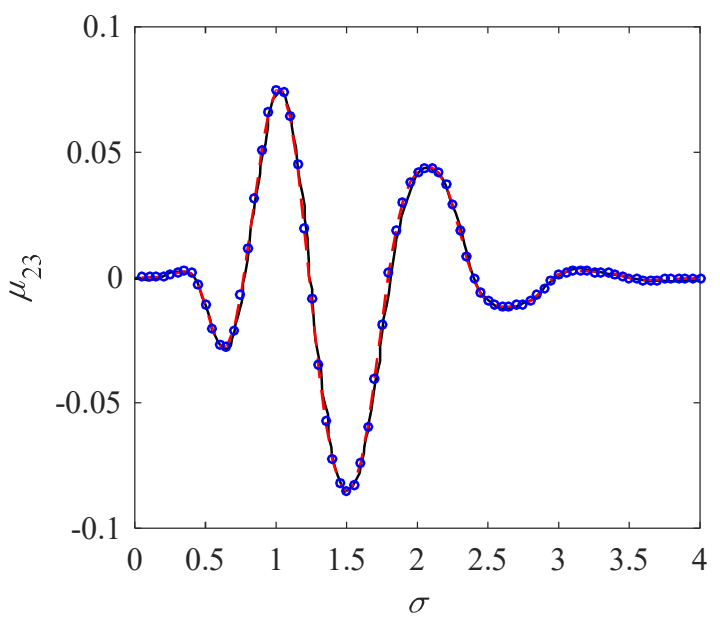

$(f)$

Fig. 2. Added mass of a submerged elliptical cylinder. (a) sway; (b) sway-heave; $(c)$ heave; $(d)$ sway-roll; $(e)$ roll; (f) heave-roll. Solid lines: results from Fig. 4 of Sturova (2015); dashed lines: $M=70, N_{0}=90$ and $N_{F}=180$; open circles: $M=100, N_{0}=180$ and $N_{F}=360 . \quad\left(a=1, \quad b=0.5, \quad\left(x^{\prime}, z^{\prime}\right)=(0,-1), \quad H=25\right.$, $x_{1}=-x_{2}=-2.5, \quad h_{1}=0.025$ and $h_{2}=0.1, d_{1}=0$ and $d_{2}=0, m_{1}=0.0225$ and $m_{2}=0.09, L_{1}=0.0356$ and $L_{2}=2.2791$ )

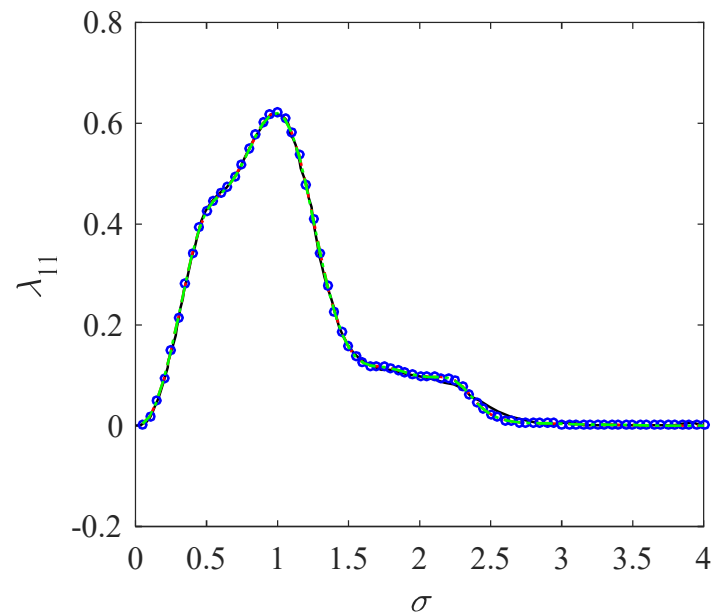

(a)

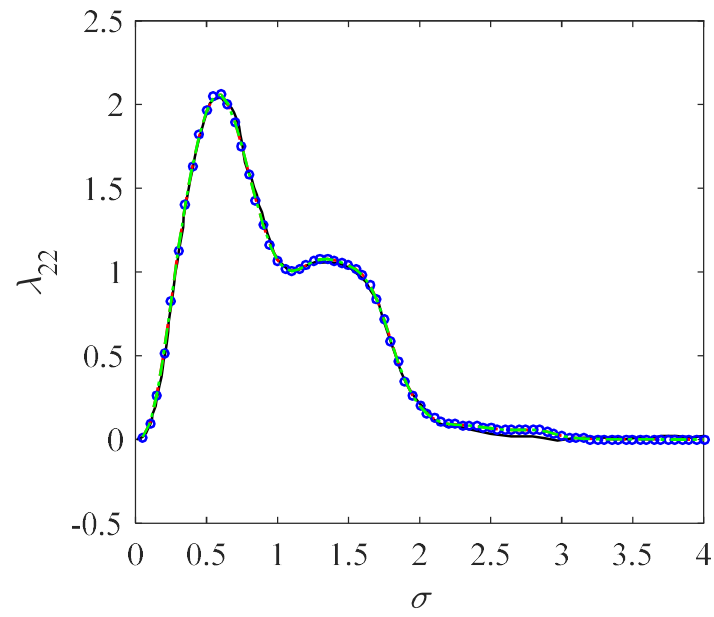

(c)

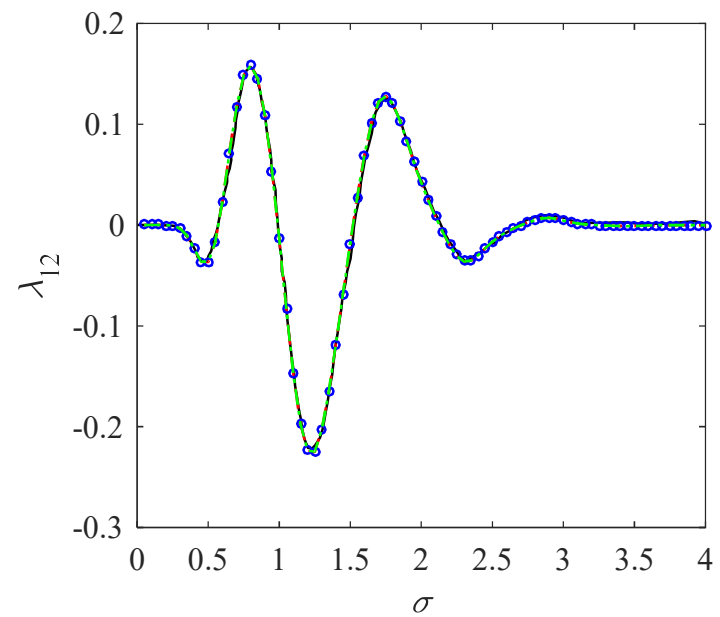

(b)

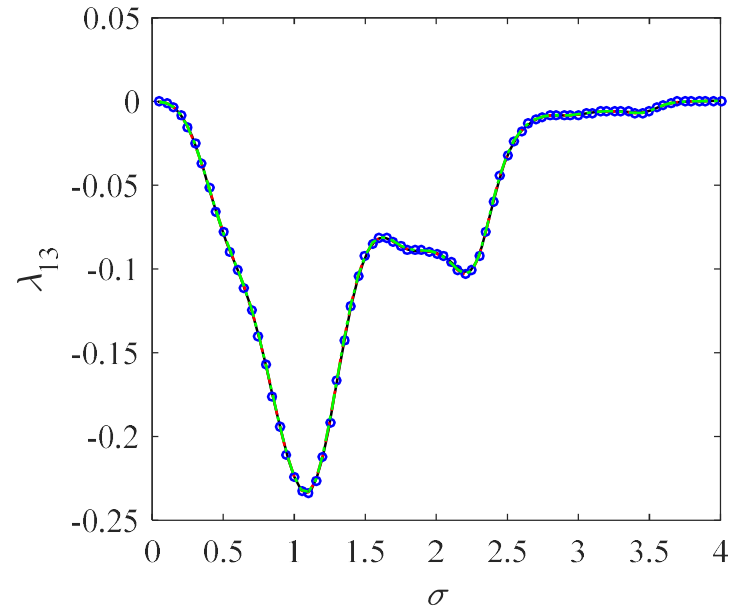

(d) 


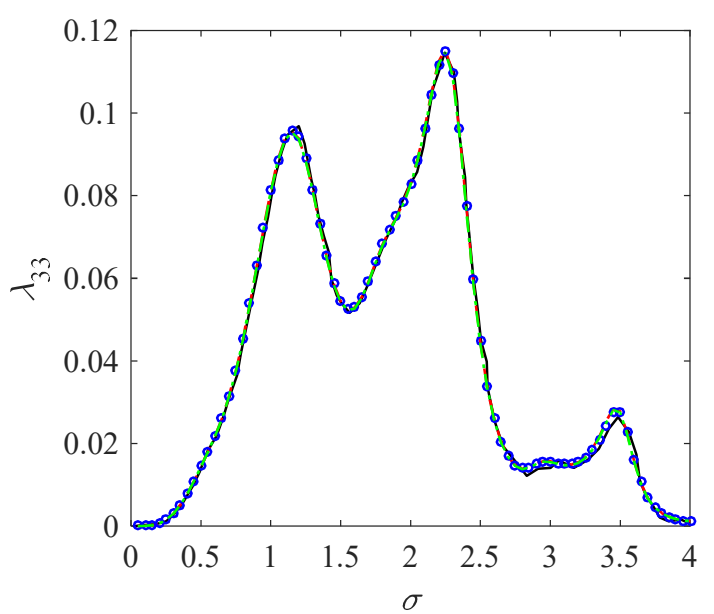

(e)

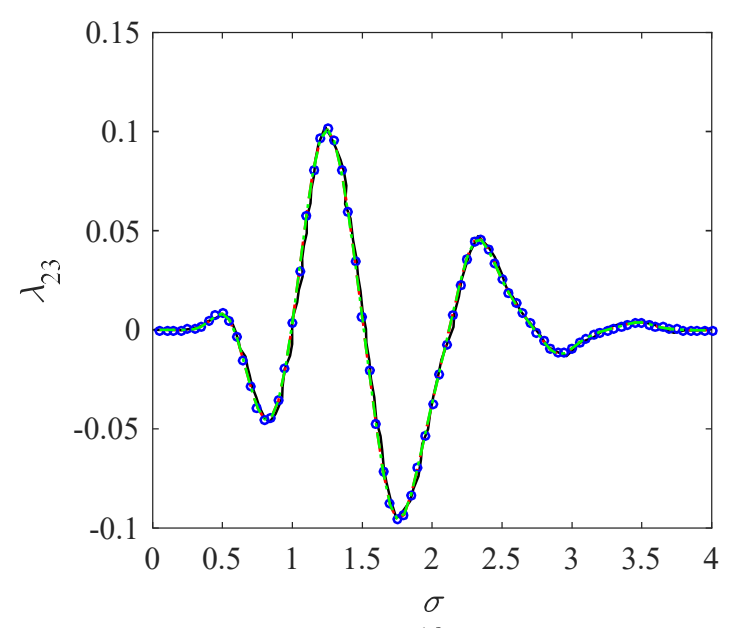

$(f)$

Fig. 3. Damping coefficient of an elliptical cylinder. $(a)$ sway; $(b)$ sway-heave; $(c)$ heave; $(d)$ sway-roll; $(e)$ roll; $(f)$ heave-roll. Solid lines: results from Fig. 5 of Sturova (2015); dashed lines: $M=70, N_{0}=90$ and $N_{F}=180$; dash-dotted lines: same to dashed lines, but by the far field formula; open circles: $M=100, N_{0}=180$ and $N_{F}=360 .\left(a=1, b=0.5,\left(x^{\prime}, z^{\prime}\right)=(0,-1), H=25, x_{1}=-x_{2}=-2.5, h_{1}=0.025\right.$ and $h_{2}=0.1, d_{1}=0$ and $d_{2}=0, m_{1}=0.0225$ and $m_{2}=0.09, L_{1}=0.0356$ and $\left.L_{2}=2.2791\right)$

\subsubsection{A floating cylinder}

We further choose the case of a body floating on the water surface confined between two semi-infinite ice sheets. The semi-analytical solutions of this problem for a rectangular body of width $a$ and draught $b$ have been obtained by Ren et al. (2016) through the eigenfunction expansion method. The same parameters in their Fig. 3 are adopted here. The added mass and damping coefficient with different $M, N_{0}$ and $N_{F}$ are presented in Fig. 4 and Fig. 5 respectively, while the corresponding wave exciting force is shown in Fig. 6. The damping coefficients and wave exciting forces are also computed by the far field formula (see Eqs. (B2) and (B5) of Ren et al. (2016)). We notice that $\mu_{j k}$ and $\lambda_{j k}$ are zero for the coupling terms between the symmetric and antisymmetric modes, as the body is symmetric with respect to $x=0$, and these zero terms are omitted. It can be seen from these figures that the results obtained with $M=70, \quad N_{0}=90, \quad N_{F}=180$ and $M=100, \quad N_{0}=180, \quad N_{F}=360$ are graphically indistinguishable, and both the near field and the far field formula give the same results. Comparison with the semi-analytical solutions of Ren et al. (2016) also shows an excellent agreement. This once again confirms that the present hybrid method is effective and accurate for the interaction problem of waves with a body floating on polynya. 


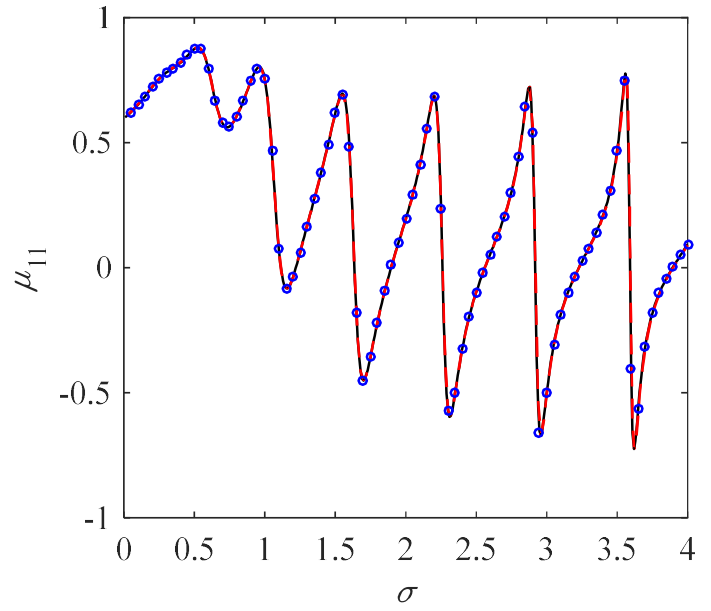

(a)

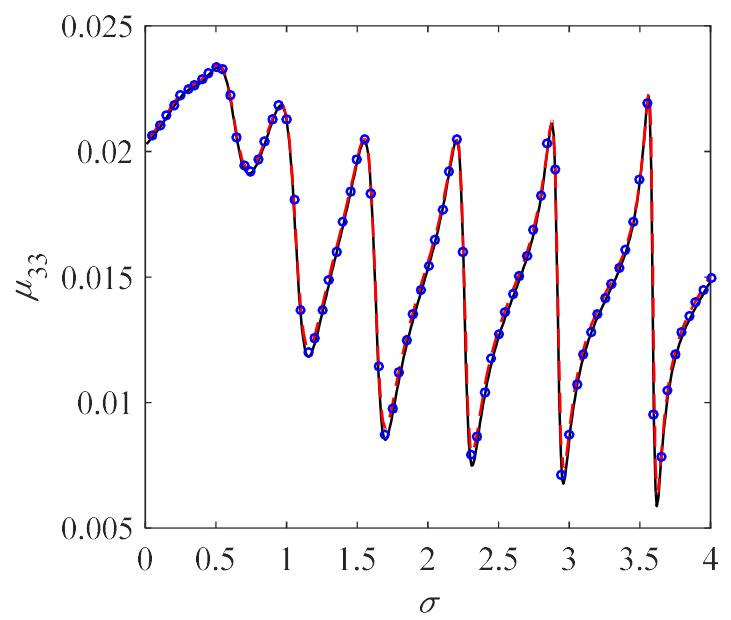

(c)

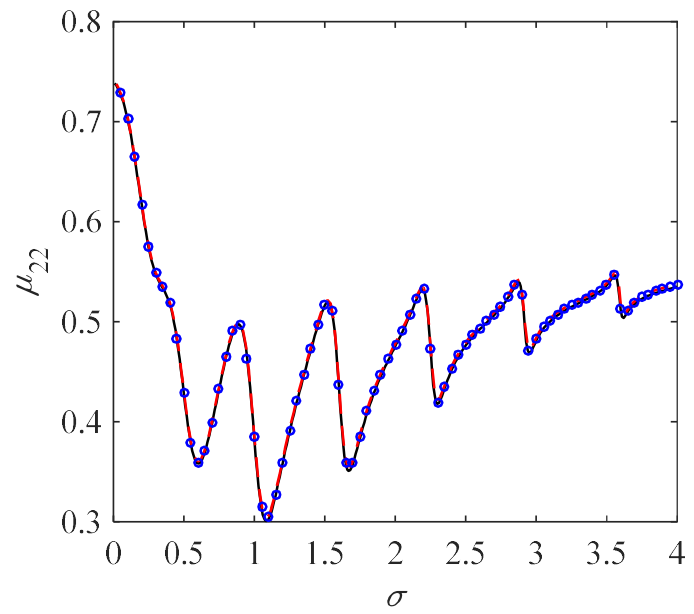

(b)

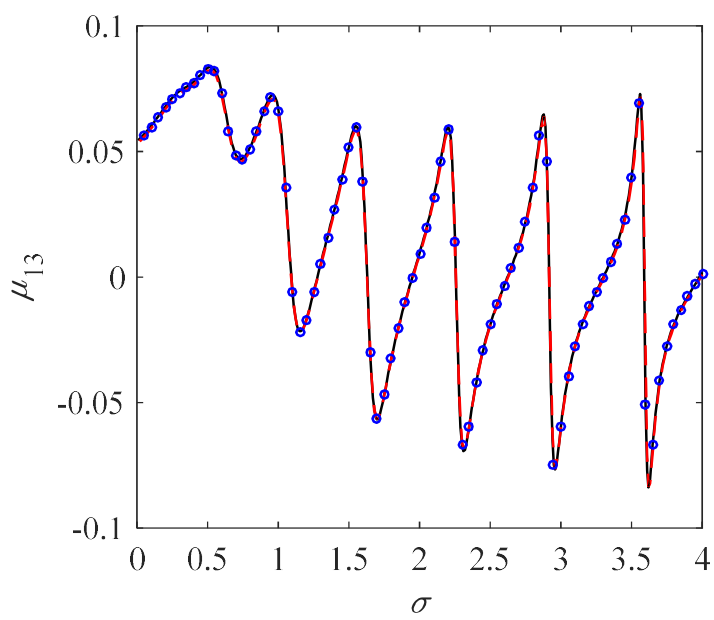

(d)

Fig. 4. Added mass of a floating rectangular body. (a) sway; (b) heave; (c) roll; (d) sway-roll or roll-sway. Solid lines: results from Fig. 3 of Ren et al. (2016); dashed lines: $M=70, N_{0}=90$ and $N_{F}=180$; open circles: $M=100, \quad N_{0}=180$ and $N_{F}=360 . \quad\left(a=1, \quad b=0.5, \quad\left(x^{\prime}, z^{\prime}\right)=(0,-b / 2), \quad H=10, \quad x_{1}=-x_{2}=-5\right.$, $\left.h_{1}=h_{2}=0.1, d_{1}=d_{2}=0.09, m_{1}=m_{2}=0.09, \quad L_{1}=L_{2}=4.5582\right)$

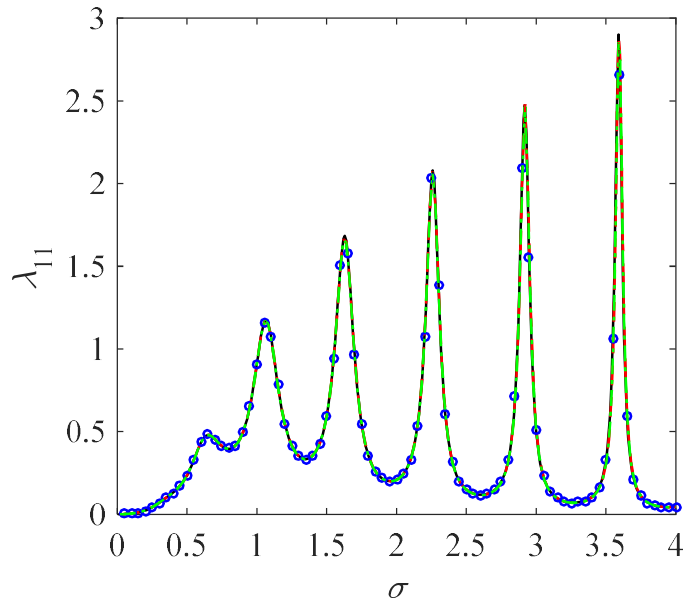

(a)

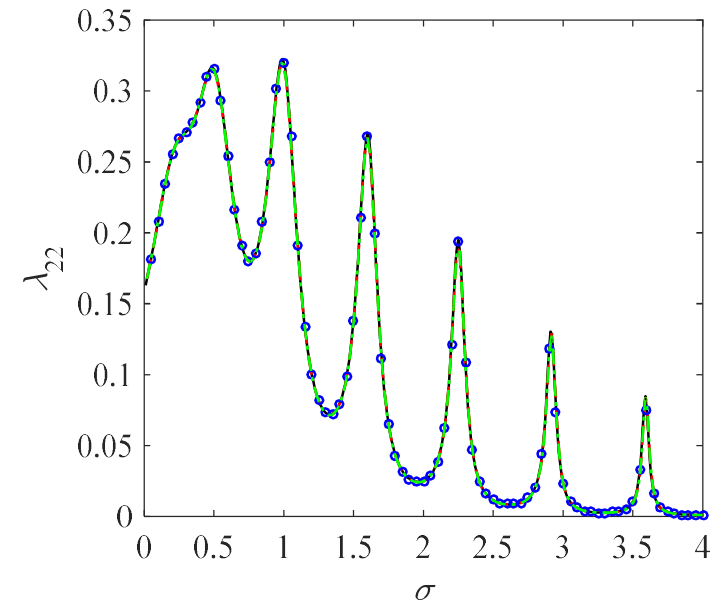

(b) 


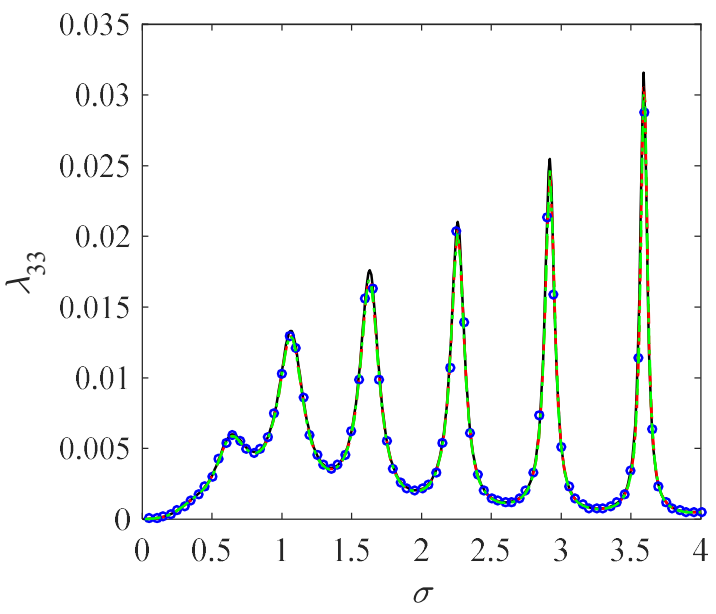

(c)

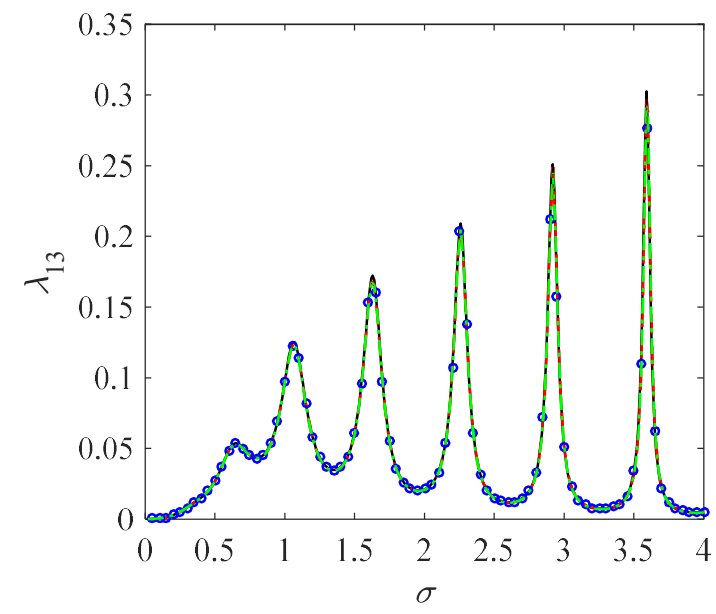

(d)

Fig. 5. Damping coefficient of a floating rectangular body. (a) sway; $(b)$ heave; $(c)$ roll; $(d)$ sway-roll or roll-sway. Solid lines: results from Fig. 3 of Ren et al. (2016); dashed lines: $M=70, N_{0}=90$ and $N_{F}=180$; dash-dotted lines: same to dashed lines, but by the far field formula; open circles: $M=100, N_{0}=180$ and $N_{F}=360$. $\left(a=1, b=0.5, \quad\left(x^{\prime}, z^{\prime}\right)=(0,-b / 2), H=10, x_{1}=-x_{2}=-5, \quad h_{1}=h_{2}=0.1, d_{1}=d_{2}=0.09, \quad m_{1}=m_{2}=0.09\right.$ ， $L_{1}=L_{2}=4.5582$ )

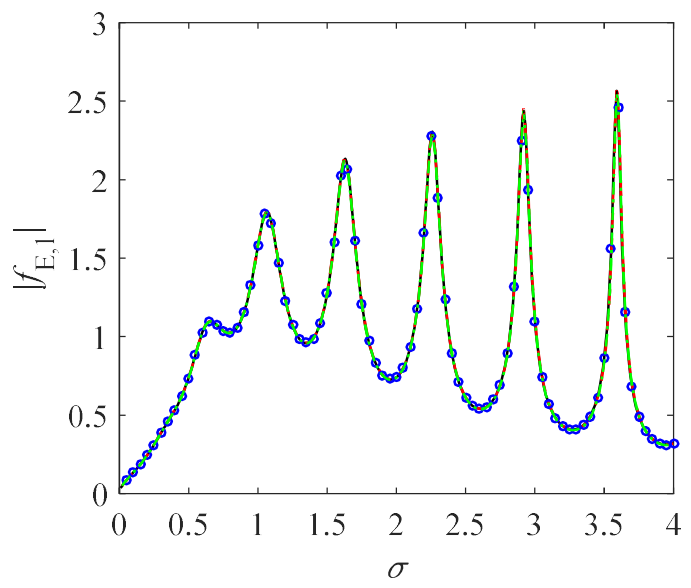

(a)

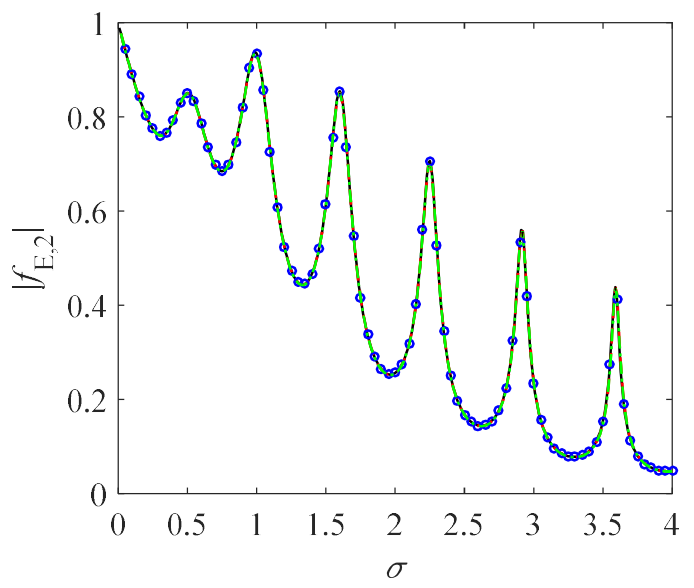

(b)

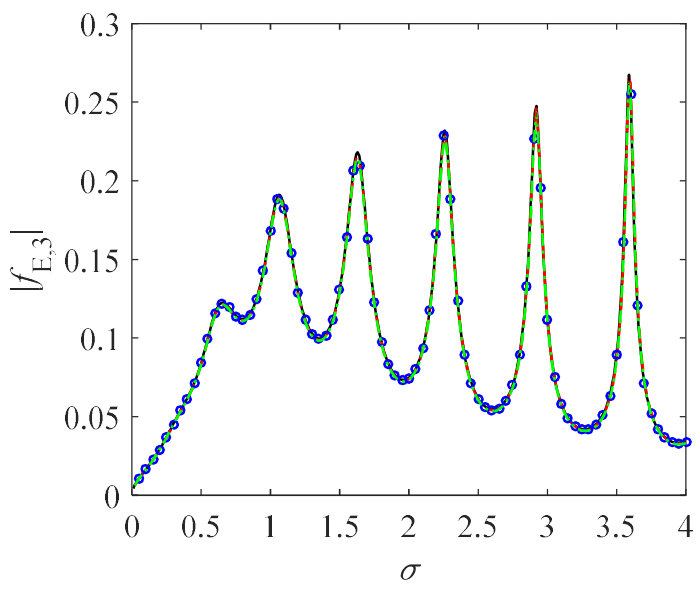

(c) 
Fig. 6. Wave exciting force on a floating rectangular body. (a) force in sway mode; $(b)$ force in heave mode; $(c)$ force in roll mode. Solid lines: results from Fig. 6 of Ren et al. (2016); dashed lines: $M=70, N_{0}=90$ and $N_{F}=180$; dash-dotted lines: same to dashed lines, but by the far field formula; open circles: $M=100$, $N_{0}=180$ and $N_{F}=360 . \quad\left(a=1, b=0.5, \quad\left(x^{\prime}, z^{\prime}\right)=(0,-b / 2), \quad H=10, \quad x_{1}=-x_{2}=-5, \quad h_{1}=h_{2}=0.1\right.$, $\left.d_{1}=d_{2}=0.09, \quad m_{1}=m_{2}=0.09, L_{1}=L_{2}=4.5582\right)$

\subsection{Hydrodynamic force and motion response of a floating elliptical cylinder}

We now carry out the simulations for the motion of a floating body excited by an incident wave to investigate the effects of ice draught and body shape. The body shape is a semi elliptical cylinder defined by $x^{2} /(a / 2)^{2}+z^{2} / b^{2}=1$ and its mass centre and rotational centre are both assumed to be at the buoyancy centre, i.e. $\left(x^{\prime}, z^{\prime}\right)=(0,-4 \mathrm{~b} / 3 a \pi)$. The body mass $m_{11}=m_{22}=m=\pi b / 4 a$, $m_{33}$ is the moment of inertia of the body. The non zero hydrostatic restoring coefficients are $C_{22}=1$ and $C_{33}=1 / 12$ respectively. The remaining terms of $m_{i j}$ and $C_{i j}$ in Eq. (39) are equal to zero. The following results are obtained with $M=70, N_{0}=180$ and $N_{F}=360$.

\subsubsection{The effects of ice draught on hydrodynamic force}

We first investigate the effects of ice draught on the hydrodynamic force exerted on the floating cylinder. The dimensionless added mass and damping coefficients are shown in Fig. 7 and Fig. 8 respectively, while the wave exciting force is presented in Fig. 9. We can observe that at very low wave number, there is no visible difference between these two sets of results. In fact, as the wavelength is very large, the draught of the ice is then relatively small and its effect is therefore far less significant. However, these two sets of results are not mathematically identical, as the locations of the ice sheet surfaces are different, on which the boundary condition is imposed. Differences between these two sets of results appear when the wave number increases. We can also see that the extreme values of the hydrodynamic coefficients with nonzero ice draughts are much larger compared to those with zero ice draughts as $\sigma>2$.

Some of the features can be explained through the far field formula for the damping coefficient and wave exciting force (Ren et al., 2016). We have in the dimensional form

$$
\lambda_{k j}=\rho \omega\left(Q_{0}^{(1)} C_{g}^{(1)} R_{k, 0}^{*} R_{j, 0}+Q_{0}^{(2)} C_{g}^{(2)} T_{k, 0}^{*} T_{j, 0}\right),(k, j=1,2,3)
$$

and

$$
f_{E, j}=-2 \mathrm{i} \rho g R_{j, 0} C_{g}^{(1)} Q_{0}^{(1)},(j=1,2,3)
$$

where

$$
Q_{0}^{(j)}=\frac{\rho \omega\left[L_{j}\left(\kappa_{0}^{(j)}\right)^{4}+\rho g\right]}{\left[L_{j}\left(\kappa_{0}^{(j)}\right)^{4}+\rho g-m_{j} \omega^{2}\right]^{2}},(j=1,2)
$$

and $C_{g}^{(j)}$ is the group velocity of the propagating wave underneath the ice in region $\Omega_{j}$, and can 
be given as

$$
C_{g}^{(j)}=\frac{d \omega}{d\left(-\mathrm{i} \kappa_{0}^{(j)}\right)}=\mathrm{i} \frac{\frac{\omega}{2 \kappa_{0}^{(j)}}\left(1+\frac{2 \kappa_{0}^{(j)}\left(H-d_{j}\right)}{\sin \left[2 \kappa_{0}^{(j)}\left(H-d_{j}\right)\right]}\right)+\frac{2 L_{j}\left(\kappa_{0}^{(j)}\right)^{3} \omega}{L_{j}\left(\kappa_{0}^{(j)}\right)^{4}+\rho g-m_{j} \omega^{2}}}{\frac{L_{j}\left(\kappa_{0}^{(j)}\right)^{4}+\rho g}{L_{j}\left(\kappa_{0}^{(j)}\right)^{4}+\rho g-m_{j} \omega^{2}}},(j=1,2)
$$

Here the notation $j$ in Eqs. (44) and (45) represents the ice covered regions, while in Eqs. (42) and (43) it represents the motion modes. Invoking Eq. (14), we have that $Q_{0}^{(j)}>0, C_{g}^{(j)}>0$, and therefore $\lambda_{k k}>0$. When there is no ice, both $C_{g}^{(j)}$ and Eq. (42) will become those well known results in open water (e.g. Eq. (8.6.11) of Mei et al. (2005)).

We notice that Eq. (43) is for the wave from $x=-\infty$, for which $f_{E, j}$ could be marked by a superscript - . For the wave from $x=+\infty$, this equation can be rewritten as

$$
f_{E, j}^{+}=-2 \mathrm{i} \rho g T_{j, 0} C_{g}^{(2)} Q_{0}^{(2)}
$$

Substituting Eqs. (43) and (46) into (42), we obtain the link between the damping coefficient and excitation force

$$
\lambda_{k j}=\frac{\omega}{4 \rho g^{2}}\left(\frac{\left(f_{E, k}^{-}\right)^{*}\left(f_{E, j}^{-}\right)}{C_{g}^{(1)} Q_{0}^{(1)}}+\frac{\left(f_{E, k}^{+}\right)^{*}\left(f_{E, j}^{+}\right)}{C_{g}^{(2)} Q_{0}^{(2)}}\right)
$$

which can be found also to become the well known results in open water when there is no ice. We notice that $\left|R_{k, 0}\right|=\left|T_{k, 0}\right|, \quad C_{g}^{(1)}=C_{g}^{(2)}=C_{g}$ and $Q_{0}^{(1)}=Q_{0}^{(2)}=Q_{0}$ when $d_{1}=d_{2}=d$, since the problem is either symmetric or anti symmetric about $x=0$. The above equations could be further simplified.

It can be seen from Fig. 8 and Fig. 9 that as $\omega \rightarrow 0$, the damping coefficient and wave exciting force tend to zero, apart from that in the heave mode. In fact, from Eqs. (3) and (4), we can see that as $\omega \rightarrow 0$ the conditions on the free surface and ice sheet will become $\partial \phi_{k} / \partial z=\mathrm{O}\left(\omega^{2}\right)$. Appling the Gauss theorem to the boundary $S$ of the entirely fluid domain $V$, we have

$$
\oint_{S} \frac{\partial \phi_{k}}{\partial n} \mathrm{~d} S=\int_{V} \nabla^{2} \phi_{k} \mathrm{~d} V=0
$$

Removing the bottom surface where $\partial \phi_{k} / \partial n=0$, we then have

$$
\int_{S_{+\infty}} \frac{\partial \phi_{k}^{(2)}}{\partial x} \mathrm{~d} S-\int_{S_{-\infty}} \frac{\partial \phi_{k}^{(1)}}{\partial x} \mathrm{~d} S=-\int_{S_{0}} \frac{\partial \phi_{k}^{(3)}}{\partial n} \mathrm{~d} S+\mathrm{O}\left(\omega^{2}\right)
$$

The body surface boundary condition in Eq. (6) indicates that the right hand side of the above equation is zero for sway and roll, and is equal to $a$ for heave. Invoking Eqs. (10) and (11), on the left hand side of Eq. (49) as $\omega \rightarrow 0$, we have

$$
-T_{k, 0} \kappa_{0}^{(2)}\left(H-d_{2}\right)-R_{k, 0} \kappa_{0}^{(1)}\left(H-d_{1}\right)=-a \delta_{k, 2}+\mathrm{O}\left(\omega^{2}\right)
$$

As $\omega \rightarrow 0$, Eq. (14) becomes

$$
\omega^{2} /\left(\kappa_{m}^{(j)}\right)^{2}=-\left(H-d_{j}\right) g
$$

and 


$$
\begin{gathered}
Q_{0}^{(j)}=\frac{\omega}{g} \\
C_{g}^{(j)}=\sqrt{\left(H-d_{j}\right) g}
\end{gathered}
$$

Using the above results in Eqs. (42) and (43), we have at $\omega=0$

$$
\lambda_{22}=\frac{\rho a^{2} \sqrt{g /(H-d)}}{2}
$$

and

$$
f_{E, 2}=-\rho g a
$$

while other terms in $\lambda_{k j}$ and $f_{E, k}$ are equal to zero. In the dimensionless form $\lambda_{22} /\left(\rho a^{2} \sqrt{g / a}\right)=\sqrt{a /\left(H-d_{1}\right)} / 2$ and $\left|f_{E, 2}\right| /(\rho g a)=1$. They are consistent with those in Fig. $8(b)$ and Fig. 9 (b) respectively, as well as those previous results in Fig. 5 (b) and Fig. $6(b)$.

Eq. (54) also indicates that when others remain the same, the damping coefficient may increase when $d$ increases. This is closely related to the fact that $R_{k, 0}$ increases when $d$ increases, as shown in Eq. (50) (notice $\left|R_{k, 0}\right|=\left|T_{k, 0}\right|$ in the current case). The relative difference between draughts in the two cases considered in Fig. 8 and Fig. 9 is only $0.9 \%$. Therefore, the difference between the results in these two cases is generally small. However, their difference is quite significant at the peaks or troughs of the curves. This is expected when the slope of the curve is nearly vertical. A small variation of the configuration of the problem can lead to a major difference in the results. To reflect this further $\left|R_{k, 0}\right|$ is plotted in Fig. 10 against $\sigma$. Its oscillation with $\sigma$ as well as the effect of $d$ are closely linked to those in Fig. 8 and Fig. 9 because of Eqs. (42) and (43). We also plot the transmission coefficient $\left|T_{0,0}\right|$, for wave propagation across polynya with the floating body, in Fig. 11 against $\sigma$. The reflection coefficient $\left|R_{0,0}\right|$ can be obtained directly according to the energy balance relation, e.g. Eq. (A2) in Ren et al. (2016). From this figure, it can be seen that the local extremum of $\left|T_{0,0}\right|$ for nonzero ice draught is smaller than that for zero one when $\sigma<2$, but for a larger $\sigma$ the former is much larger than the latter. This trend coincides with that for the hydrodynamic force. The comparison of Fig. 11 to Figs. 7-9 shows that for when $\sigma>2$, the abscissa of the local extremum of the hydrodynamic force is very close to that of $\left|T_{0,0}\right|$ for both zero and nonzero ice draught.

For some damping coefficients $\lambda_{k j}$, it can be seen from Fig. 8 that in the computed wave frequency range, the local extremum of $\lambda_{k j}$ increases with $\sigma$. Similar phenomenon can be also observed in Fig. 5 for the floating rectangle body. It is well known that in open water, near zero frequency $\lambda_{k j}$ generally increases with $\sigma$ as the wave is being generated and propagating outwards. $\lambda_{k j}$ will reach a peak and then it will decrease as $\sigma$ further increases, and will become zero as $\sigma \rightarrow \infty$. In polynya, we can see that the trend of the mean of the damping coefficient curve follows that in the open water. Therefore its local extremum increases within the 
certain range of $\sigma$. However, for the limiting case

$\sigma \rightarrow \infty$, it will become zero.

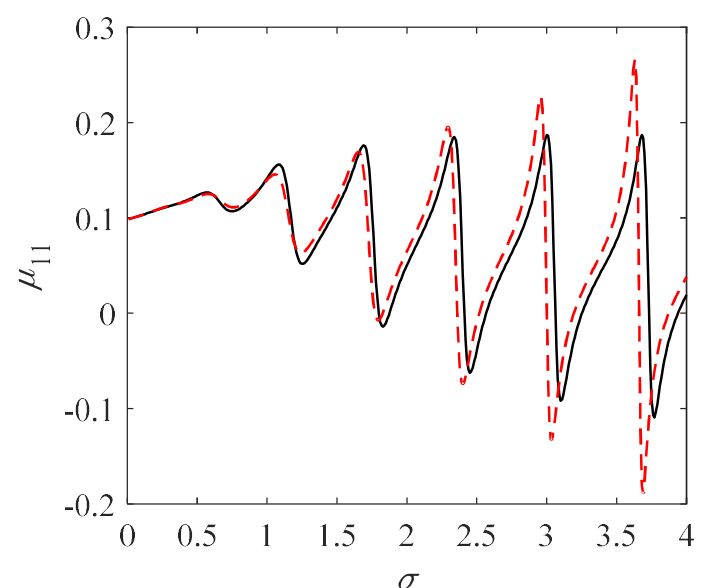

(a)

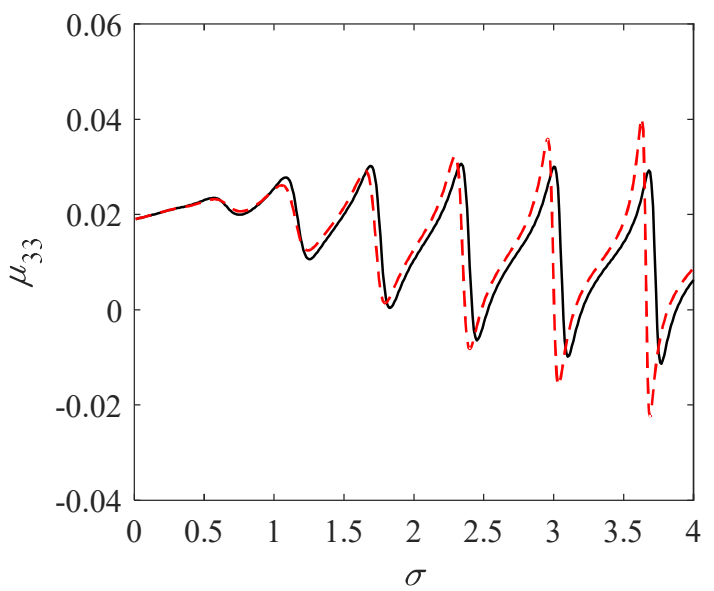

(c)

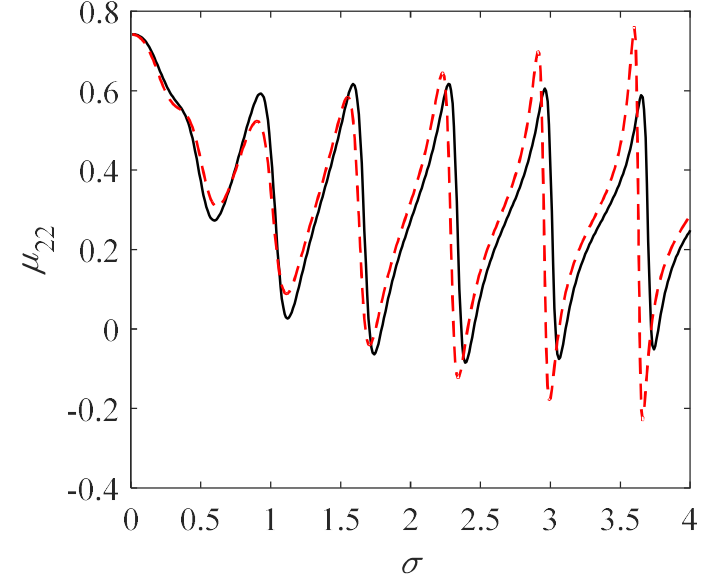

(b)

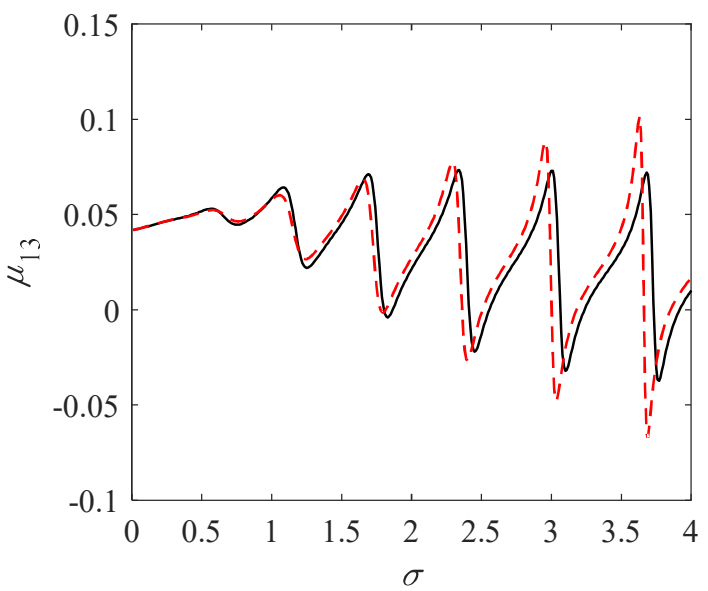

(d)

Fig. 7. Added mass against $\sigma$. (a) sway; (b) heave; $(c)$ roll; $(d)$ sway-roll or roll-sway. Solid lines: $d_{1}=d_{2}=0$; dashed lines: $d_{1}=d_{2}=0.09$. $\left(a=1, b=0.25, H=10, x_{1}=-x_{2}=-5, h_{1}=h_{2}=0.1, \quad m_{1}=m_{2}=0.09\right.$, $L_{1}=L_{2}=4.5582$ )

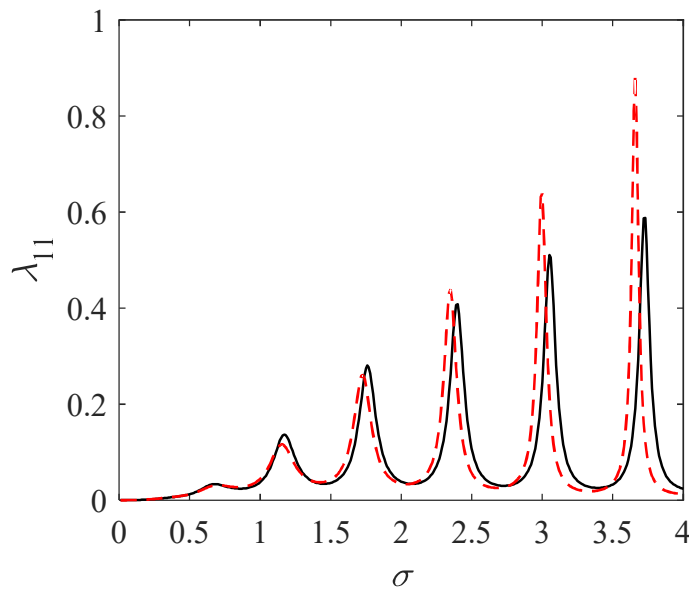

(a)

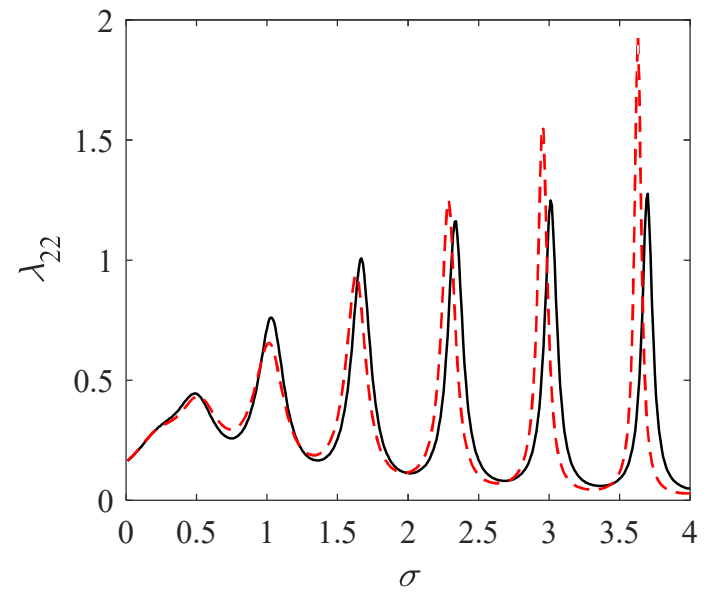

(b) 


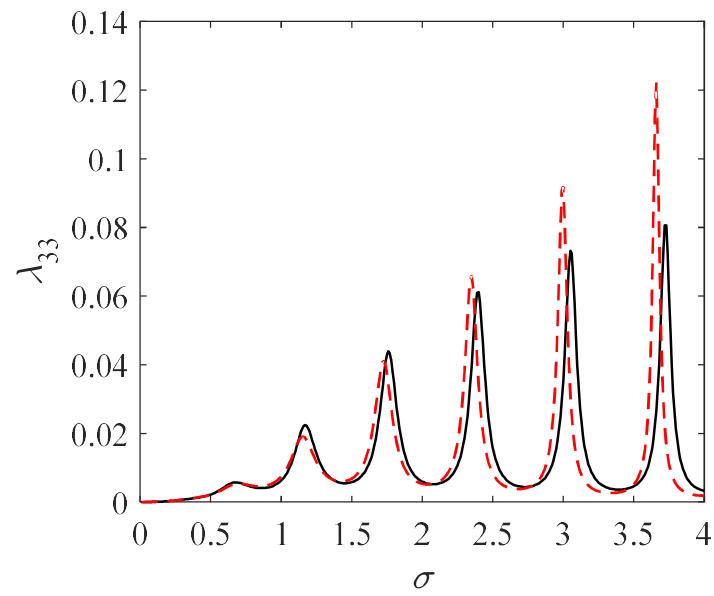

(c)

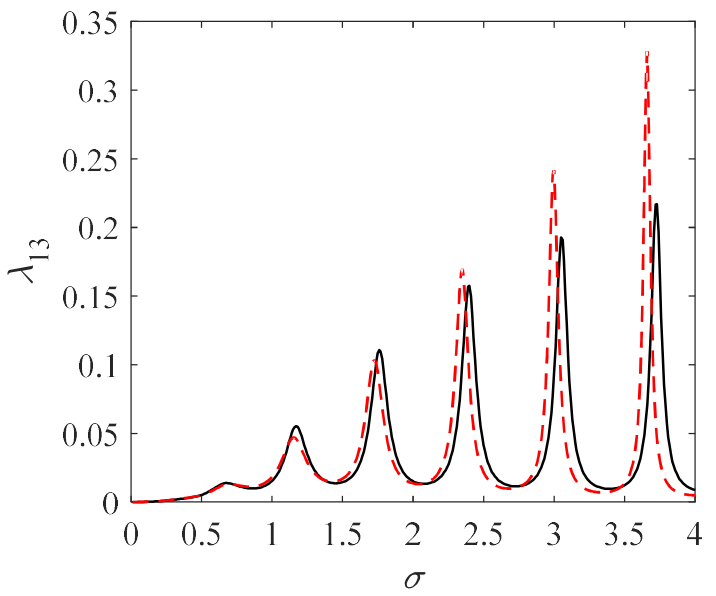

(d)

Fig. 8. Damping coefficient against $\sigma$. (a) sway; $(b)$ heave; $(c)$ roll; (d) sway-roll or roll-sway. Solid lines: $d_{1}=d_{2}=0$; dashed lines: $d_{1}=d_{2}=0.09 .\left(a=1, b=0.25, H=10, x_{1}=-x_{2}=-5, \quad h_{1}=h_{2}=0.1\right.$, $\left.m_{1}=m_{2}=0.09, \quad L_{1}=L_{2}=4.5582\right)$

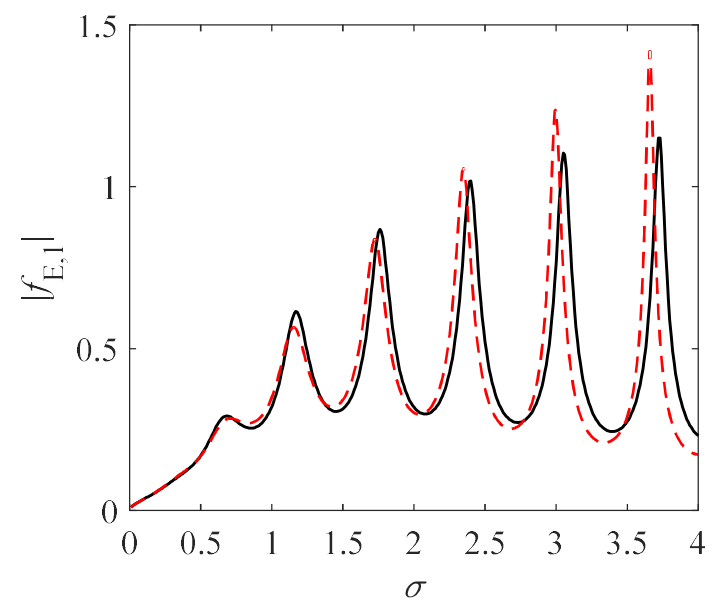

(a)

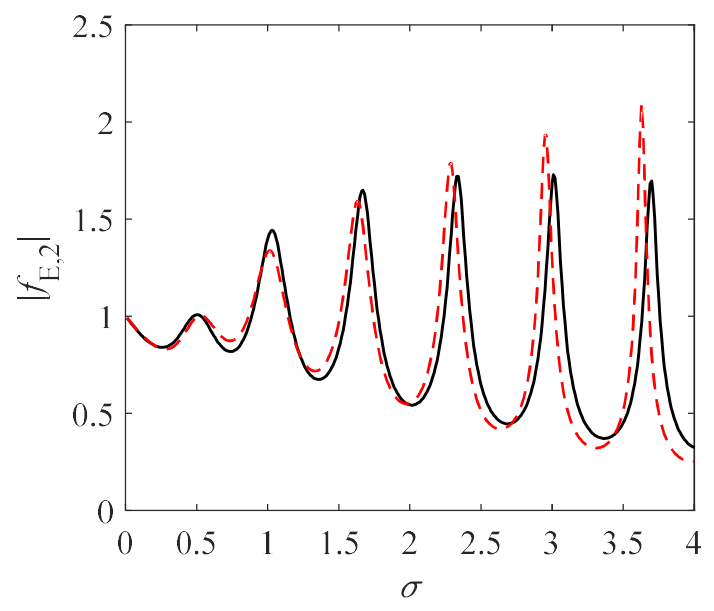

(b)

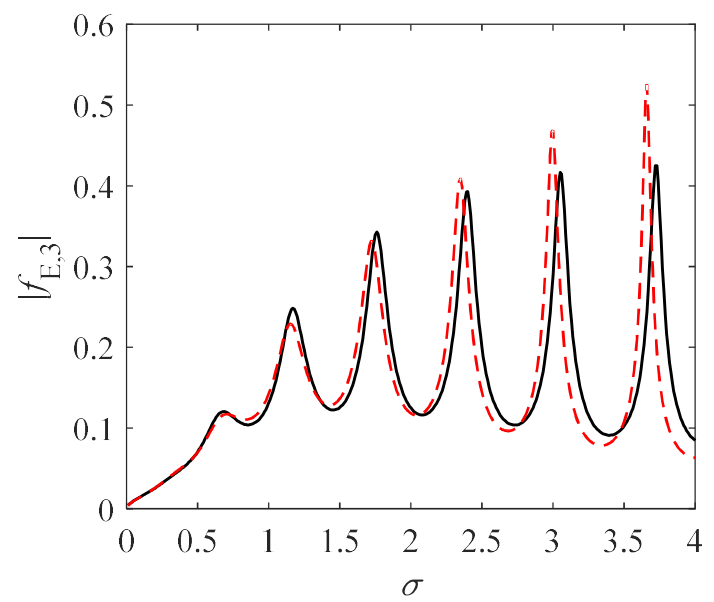

(c)

Fig. 9. Amplitude of the wave exciting force against $\sigma$. (a) force in sway mode; $(b)$ force in heave mode; $(c)$ 
force in roll mode. Solid lines: $d_{1}=d_{2}=0$; dashed lines: $d_{1}=d_{2}=0.09 .(a=1, b=0.25, H=10$, $\left.x_{1}=-x_{2}=-5, \quad h_{1}=h_{2}=0.1, \quad m_{1}=m_{2}=0.09, L_{1}=L_{2}=4.5582\right)$

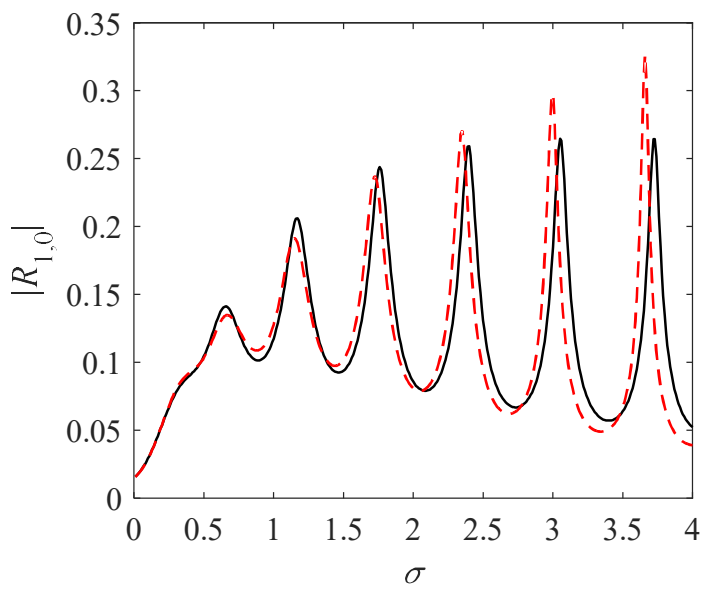

(a)

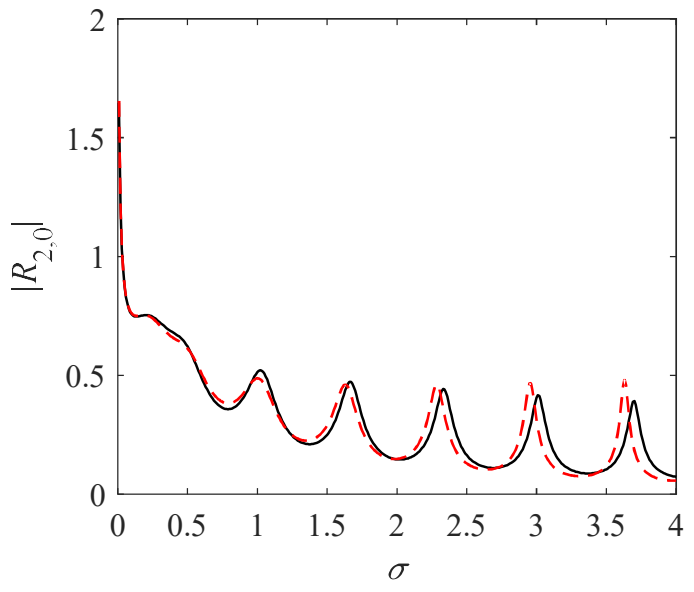

(b)

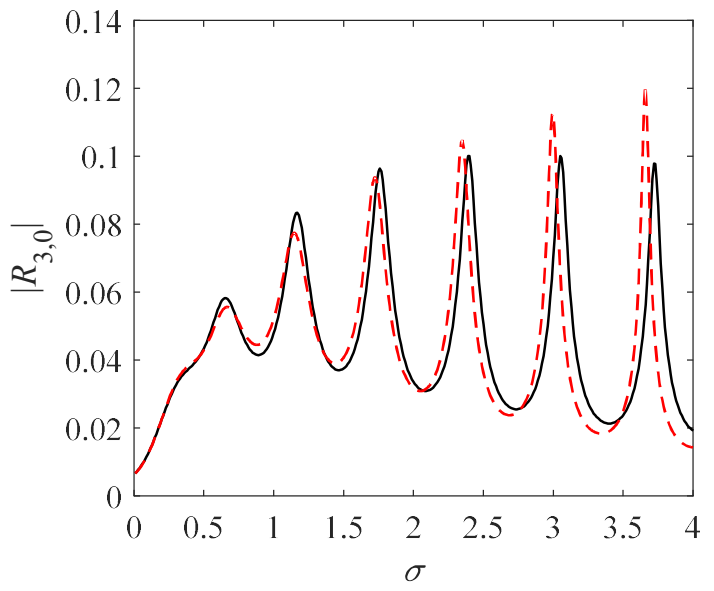

(c)

Fig. 10. $R_{j, 0}$ against $\sigma .(a) \quad j=1 ;$ (b) $j=2 ;$ (c) $j=3$. Solid lines: $d_{1}=d_{2}=0$; dashed lines: $d_{1}=d_{2}=0.09 .\left(a=1, b=0.25, H=10, x_{1}=-x_{2}=-5, h_{1}=h_{2}=0.1, m_{1}=m_{2}=0.09, L_{1}=L_{2}=4.5582\right)$

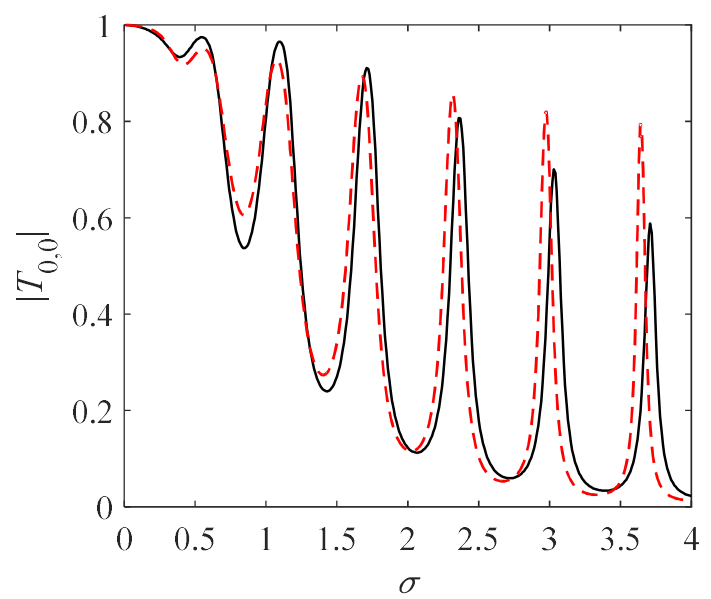

Fig. 11. $T_{0,0}$ against $\sigma$. Solid line: $d_{1}=d_{2}=0$; dashed line: $d_{1}=d_{2}=0.09 .(a=1, b=0.25, H=10$, $\left.x_{1}=-x_{2}=-5, \quad h_{1}=h_{2}=0.1, \quad m_{1}=m_{2}=0.09, \quad L_{1}=L_{2}=4.5582\right)$ 


\subsubsection{The effects of body shape on hydrodynamic force and motion response}

In the following computations, the parameters of polynya and ice sheets are chosen as $H=10$, $x_{1}=-x_{2}=-5, h_{1}=h_{2}=0.1, d_{1}=d_{2}=0.09, m_{1}=m_{2}=0.09, L_{1}=L_{2}=4.5582$. Here we maintain the width of waterline of the body with $a=1$ and assume the same rotational inertia with $m_{33}=0.05$, but will change the ratio of $b / a$. Three different body configurations are considered with $b / a=0.25,0.5,0.75$, respectively. The results for the open water case with $b / a=0.25$ are also provided as a comparison.

Fig. 12 and Fig. 13 respectively present the added mass and damping coefficient of the floating elliptical cylinders with different $b / a$ against dimensionless wave frequency, while Fig. 14 shows the corresponding wave exciting force. It is evident that for the case $b / a=0.5$ which corresponds to a circle, the fluid will not be disturbed by the roll oscillation when the rotational centre is taken at $(0,0)$. While the centre is at $\left(0, z^{\prime}\right)$ in general, we have $n_{3}=-z^{\prime} n_{1}$ for $b / a=0.5$. Therefore $\phi_{3}=-z^{\prime} \phi_{1}$, i.e. the results in roll mode can be obtained directly from those in sway mode. It can be seen that the sway added mass and damping coefficient both increase with $b / a$. This is due to the fact that at larger $b / a$, a larger force is required to accelerate the fluid or to push the fluid aside. Similarly, when there is an incoming wave, larger $b / a$ means a larger blockage in the wave path. This leads to a larger amplitude of the wave exciting force. As $\sigma \rightarrow 0$, the sway damping coefficient and exciting force become zero in all the cases for the reason discussed in the previous section. For the added mass in sway mode, we may notice that zero frequency for a semi elliptical cylinder on the surface of open water without ice sheet is equivalent to a full elliptical cylinder in an unbounded fluid domain. The added mass in such a case will be $\pi \rho b^{2} / 2$, which is $\pi(b / a)^{2} / 2$ in the dimensionless form. This is reflected in Fig. $12(a)$. However, the results are not identical because of the effect of the draught of the ice sheet. For the heave mode, i.e. $\partial \phi_{2} / \partial n=n_{2}$, the disturbance to the fluid is mainly arising from the bottom of the body if the body is wall sided at the water surface or $n_{2}=0$. This will become stronger when the draught becomes smaller. Therefore, larger hydrodynamic force in terms of added mass and damping coefficient is expected due to the fact the dimensionless width of the body remains unchanged. For the roll motion, the results include the effect of not only the variation of $b / a$ but also the variation of the rotational centre. Compared with the results from open water, the present results with ice sheets are quite oscillatory with $\sigma$. This is very much due to wave reflection and transmission between the open water region and ice covered region. A standing wave can occur near the body, as observed by Ren et al. (2016). 


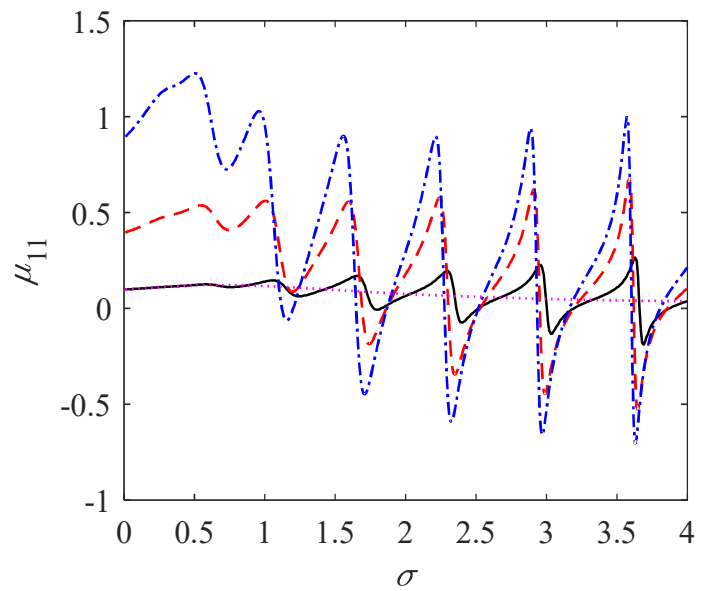

(a)

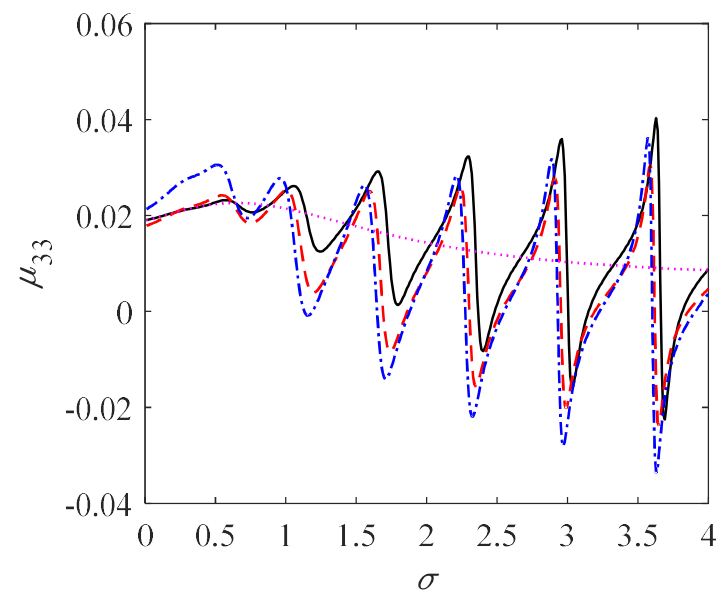

(c)

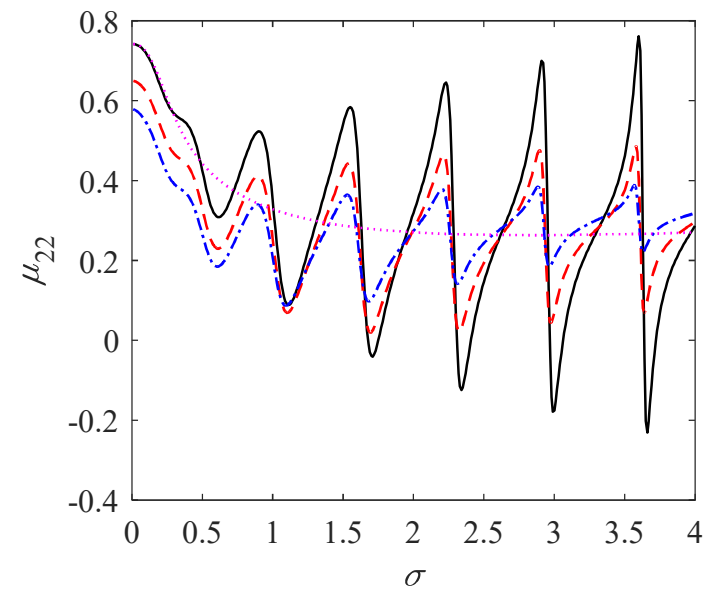

(b)

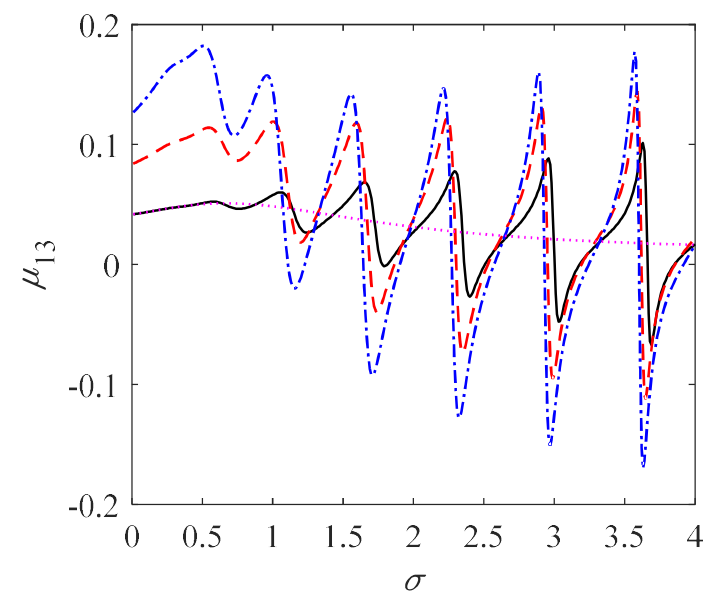

(d)

Fig. 12. Added mass against $\sigma$. (a) sway; $(b)$ heave; $(c)$ roll; $(d)$ sway-roll or roll-sway. Solid lines: $b / a=0.25$; dashed lines: $b / a=0.5$; dash-dotted lines: $b / a=0.75$; dotted lines: $b / a=0.25$, but for open water. $(a=1$, $\left.H=10, x_{1}=-x_{2}=-5, \quad h_{1}=h_{2}=0.1, d_{1}=d_{2}=0.09, m_{1}=m_{2}=0.09, \quad L_{1}=L_{2}=4.5582\right)$

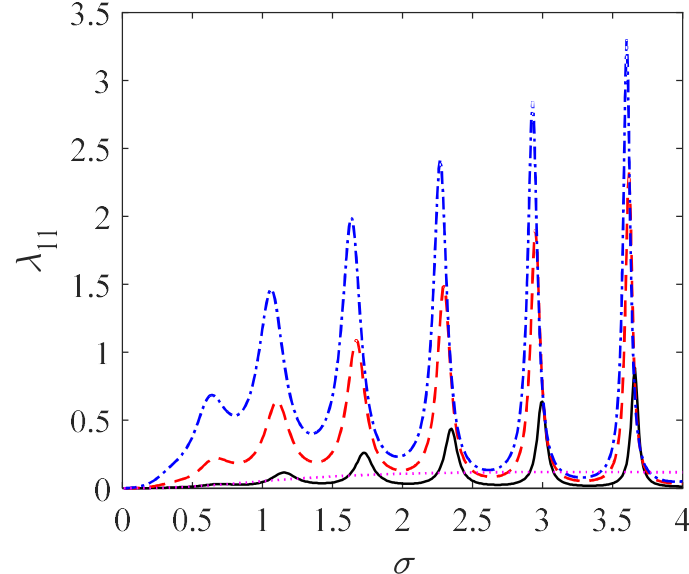

(a)

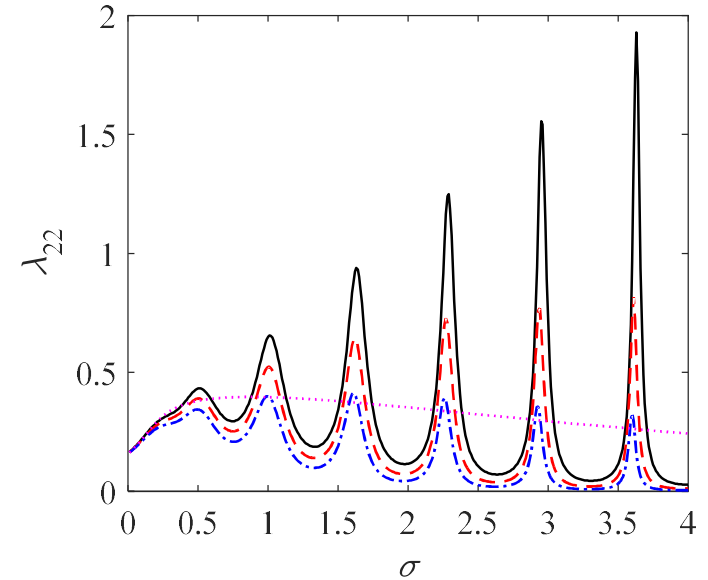

(b) 


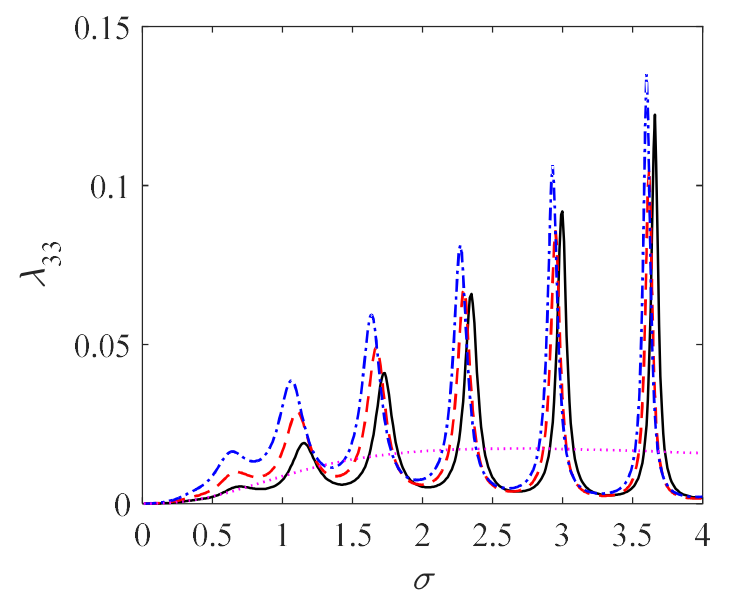

(c)

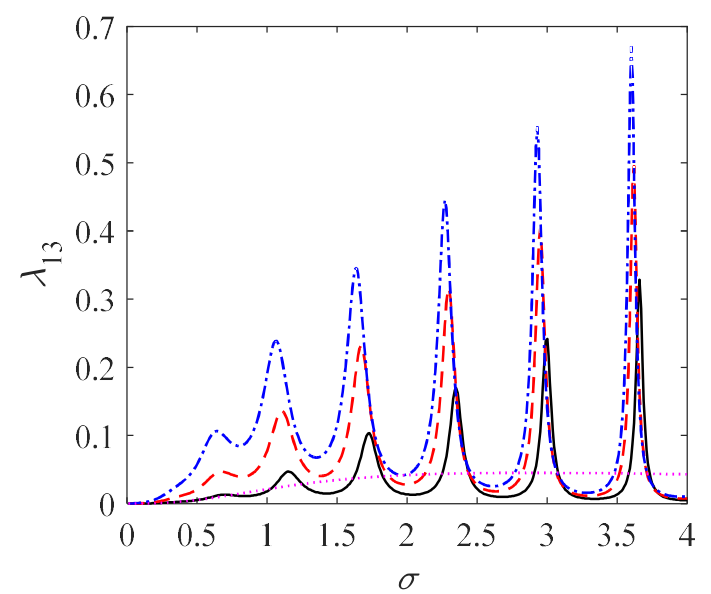

(d)

Fig. 13. Damping coefficient against $\sigma$. (a) sway; (b) heave; (c) roll; (d) sway-roll or roll-sway. Solid lines: $b / a=0.25$; dashed lines: $b / a=0.5$; dash-dotted lines: $b / a=0.75$; dotted lines: $b / a=0.25$, but for open water. $\left(a=1, H=10, x_{1}=-x_{2}=-5, h_{1}=h_{2}=0.1, d_{1}=d_{2}=0.09, m_{1}=m_{2}=0.09, L_{1}=L_{2}=4.5582\right)$

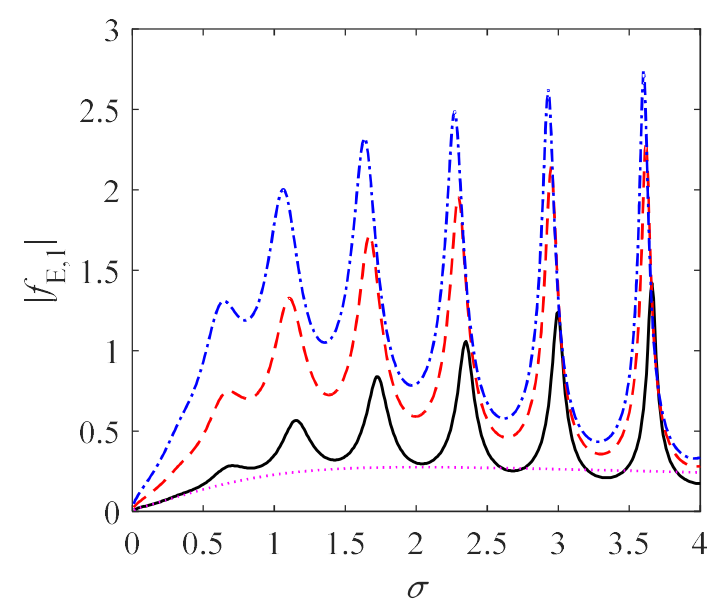

(a)

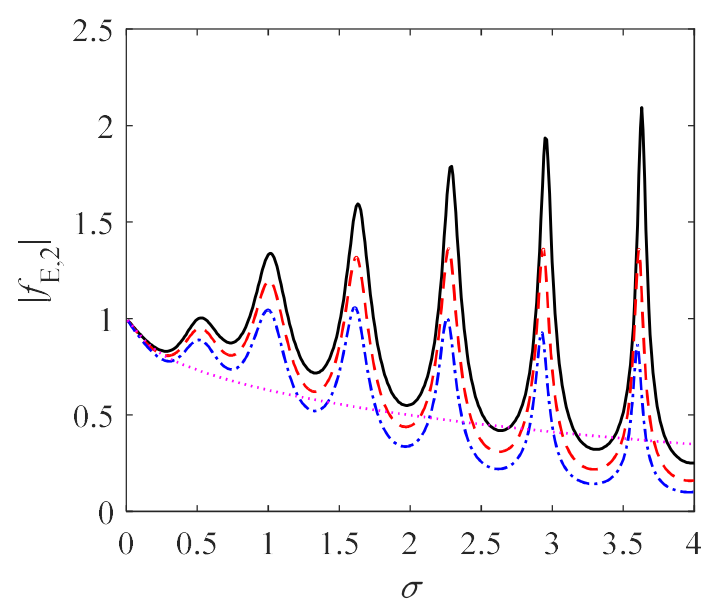

(b)

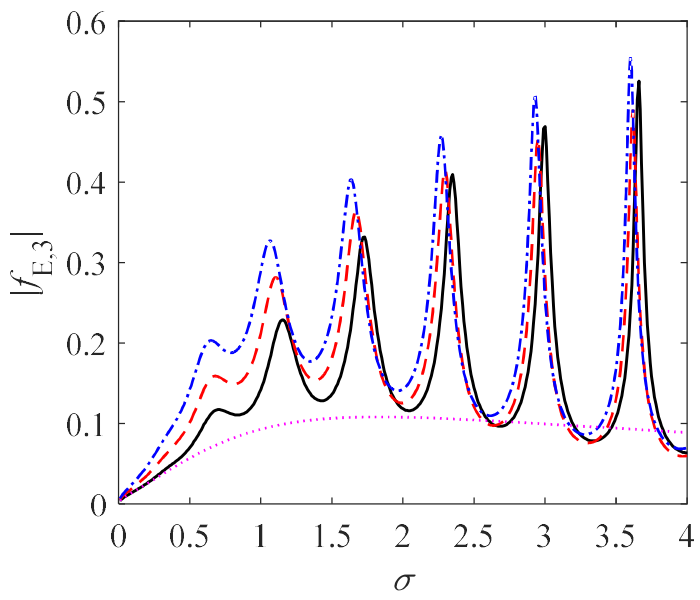

(c)

Fig. 14. Amplitude of the wave exciting force against $\sigma$. (a) force in sway mode; $(b)$ force in heave mode; $(c)$ 
force in roll mode. Solid lines: $b / a=0.25$; dashed lines: $b / a=0.5$; dash-dotted lines: $b / a=0.75$; dotted lines: $b / a=0.25$, but for open water. $\left(a=1, H=10, x_{1}=-x_{2}=-5, h_{1}=h_{2}=0.1, d_{1}=d_{2}=0.09\right.$, $\left.m_{1}=m_{2}=0.09, \quad L_{1}=L_{2}=4.5582\right)$

We then compute the motion response of the floating elliptical cylinder excited by the incident wave. The results are shown in Fig. 15, together with those from the open water $b / a=0.25$. Since the floating body is symmetric about $x=0$, the heave motion is fully decoupled from the sway and roll motions. From Eq. (39), we have in the dimensionless form

$$
\begin{aligned}
\left|\frac{\alpha_{2}}{\alpha_{0}}\right|^{2} & =\frac{\left|f_{E, 2}\right|^{2}}{\left[-\sigma\left(m_{22}+\mu_{22}\right)+C_{22}\right]^{2}+\sigma \lambda_{22}^{2}} \\
& =\frac{2 \lambda_{22} C_{g} Q_{0} / \sqrt{\sigma}}{\left[-\sigma\left(m_{22}+\mu_{22}\right)+C_{22}\right]^{2}+\sigma \lambda_{22}{ }^{2}}
\end{aligned}
$$

in which the dimensionless form of Eq. (47) and those relationships stated below Eq. (47) have been used. From Fig. $15(b)$, we can see that $\alpha_{2} / \alpha_{0}$ with different $b / a$ tends to the same value 1 when $\sigma \rightarrow 0$. This is because a body entirely follows the wave at zero frequency, to ensure the buoyancy will remain the same as the weight, as there is no dynamic effect. This can be also explained by Eq. (56) above. In fact from the dimensionless form of Eq. (55) we have $\left|f_{E, 2}\right|=\left|C_{22}\right|$, which directly gives $\left|\alpha_{2}\right| / \alpha_{0}=1$ as $\sigma \rightarrow 0$. For a simple spring system with constant mass, damping and stiffness under excitation of a periodic force at a given magnitude, the motion will be largest when the inertial term is cancelled by the restoring force term. Based on Eq. (56) above, this means that it happens when $\sigma$ satisfies

$$
f_{2}=\frac{C_{22}}{m_{22}+\mu_{22}}
$$

For the present problem $\mu_{22}$ itself is a function of $\sigma$. The curve of $f_{2}$ against $\sigma$ at each $b / a$ is plotted in Fig. $16(a)$. When it intersects with the straight line $f=\sigma$, the inertial term will be cancelled by the restoring force term and Eq. (56) will become

$$
\left|\frac{\alpha_{2}}{\alpha_{0}}\right|^{2}=\frac{\left|f_{E, 2}\right|^{2}}{\sigma\left(\lambda_{22}\right)^{2}}=\frac{2 C_{g} Q_{0}}{\sigma^{3 / 2} \lambda_{22}}
$$

From Fig. 16 (a), we can see that there are numerous intersections, for which Eq. (58) is always valid. Among all of them, the largest motion will most likely correspond to the point where the damping level will be the lowest, as the variation of $C_{g} Q_{0} / \sigma^{3 / 2}$ is much milder. This can be seen from Fig. $15(b)$, which shows that the largest heave motion appears at $\sigma=2.55,1.84,1.27$ respectively for $b / a=0.25,0.5,0.75$, which correspond to the fifth, third and first natural frequency in each case respectively. In addition to the largest peak, there are also many local peaks in the heave motion, which is very much different from the motion in the open sea without ice. A more rigorous analysis for the peaks is of course to take the derivative in Eq. (56) with respect to 
$\sigma$ and then find its zero points. The above analysis, nevertheless, shows a closer link with the undamped natural frequencies.

Sway and roll motions are both anti symmetric, and they are fully coupled. We have

$$
\begin{aligned}
& \left|\alpha_{1}\right| / \alpha_{0}=\left|\Upsilon_{1}\right| /|\Upsilon| \\
& \left|\alpha_{3}\right| / \alpha_{0}=\left|\Upsilon_{3}\right| /|\Upsilon|
\end{aligned}
$$

where

$$
\begin{aligned}
& \Upsilon_{1}= {\left[-\sigma\left(m_{33}+\mu_{33}\right)+\mathrm{i} \sqrt{\sigma} \lambda_{33}+C_{33}\right] f_{E, 1}-\left(\mathrm{i} \sqrt{\sigma} \lambda_{13}-\sigma \mu_{13}\right) f_{E, 3} } \\
& \Upsilon_{3}=\left[-\sigma\left(m_{11}+\mu_{11}\right)+\mathrm{i} \sqrt{\sigma} \lambda_{11}\right] f_{E, 3}-\left(\mathrm{i} \sqrt{\sigma} \lambda_{31}-\sigma \mu_{31}\right) f_{E, 1} \\
& \Upsilon= {\left[-\sigma\left(m_{11}+\mu_{11}\right)+\mathrm{i} \sqrt{\sigma} \lambda_{11}\right]\left[-\sigma\left(m_{33}+\mu_{33}\right)+\mathrm{i} \sqrt{\sigma} \lambda_{33}+C_{33}\right]-} \\
&\left(\mathrm{i} \sqrt{\sigma} \lambda_{13}-\sigma \mu_{13}\right)\left(\mathrm{i} \sqrt{\sigma} \lambda_{31}-\sigma \mu_{31}\right)
\end{aligned}
$$

We may notice that there is no restoring force in sway. As $\sigma \rightarrow 0$, the leading order of Eq. (63) will be $\Upsilon=\mathrm{O}(\sigma)$. From Eqs. (43), (48), (50) and (51), we have $f_{E, 1}=\mathrm{O}(\sigma)$ and $f_{E, 3}=\mathrm{O}(\sigma)$, which gives $\Upsilon_{1}=\mathrm{O}(\sigma)$ and $\Upsilon_{3}=\mathrm{o}(\sigma)$. Thus Fig. $15(a)$ shows a finite nonzero sway response at $\sigma=0$ while the roll response is zero.

For coupled motion, the natural frequency will be different from that of uncoupled motion. In the case of heave, the undamped natural frequency corresponds to the real part of the denominator of $\alpha_{2} / \alpha_{0}$ equal to zero. If we follow the same principle, the real part of the denominator $\Upsilon$ in Eq. (63) will be zero when $\sigma$ satisfies

$$
f_{1,3}=\frac{\left(m_{11}+\mu_{11}\right) C_{33}}{\left(m_{33}+\mu_{33}\right)\left(m_{11}+\mu_{11}\right)-\mu_{13} \mu_{31}}
$$

Here the relationship $\lambda_{11} \lambda_{33}=\lambda_{13} \lambda_{31}$ has been used, which can be obtained directly from Eq. (42) when $\left|R_{k, 0}\right|=\left|T_{k, 0}\right|$. The variation of $f_{1,3}$ with $\sigma$ is given in Fig. $16(b)$ for different $b / a$, together with its intersections with the straight line $f=\sigma$. We can see that the first natural frequencies for the coupled sway and roll motions at $b / a=0.25,0.5,0.75$ are $\sigma=1.36,1.43$, 1.40 respectively. The coupled motion response at each of these corresponding frequencies is much larger than those at the other intersections. The imaginary part of the denominator $\Upsilon$ is effectively the damping level. When $\operatorname{Re}(\Upsilon)=0$, we have the magnitude of the imaginary part $|\Upsilon|=0.0014,0.0032,0.0078$ for $b / a=0.25,0.5,0.75$, respectively. They are much smaller than those at the other natural frequencies for each $b / a$. 


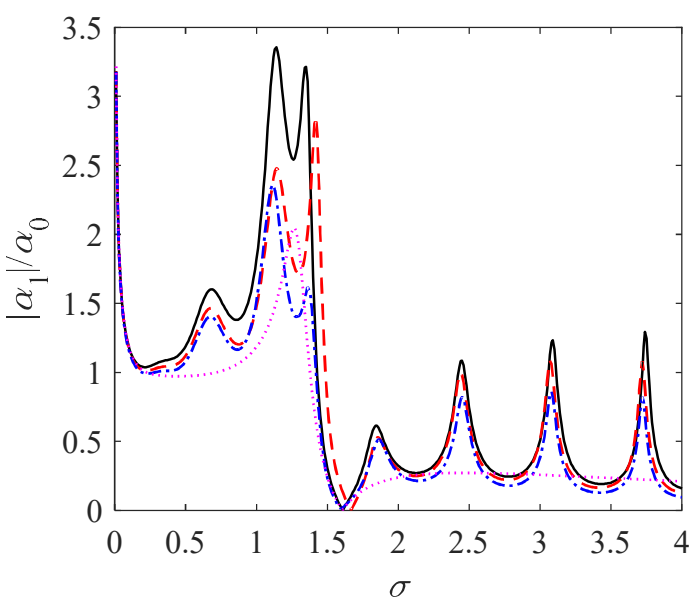

(a)

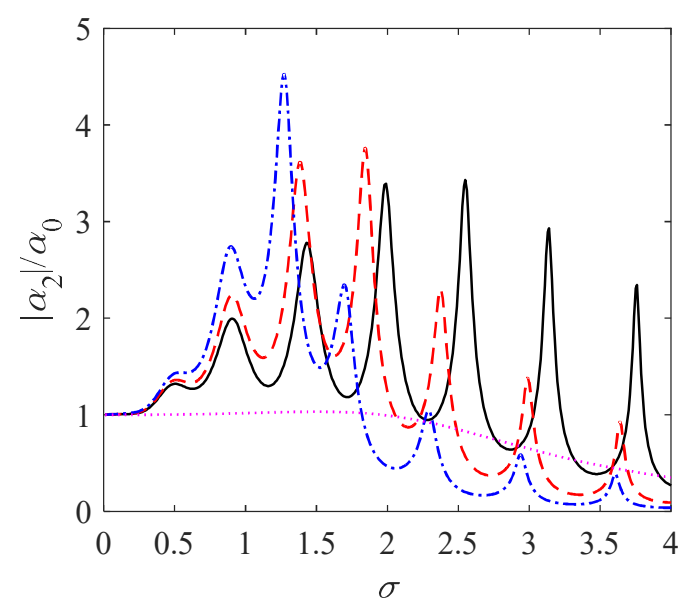

(b)

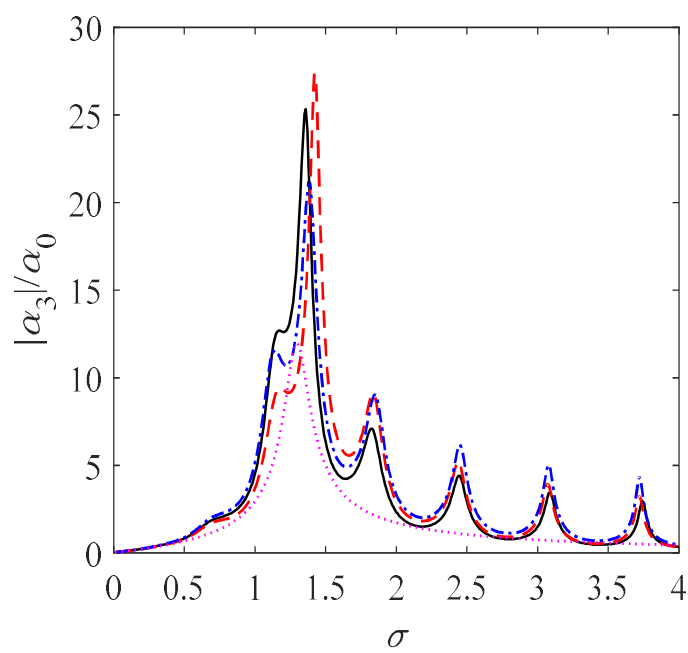

(c)

Fig. 15. Wave excited motions against $\sigma$. (a) sway; (b) heave; (c) roll. Solid lines: $b / a=0.25$; dashed lines: $b / a=0.5$; dash-dotted lines: $b / a=0.75$; dotted lines: $b / a=0.25$, but for open water. $\left(a=1, m_{33}=0.05\right.$, $\left.H=10, x_{1}=-x_{2}=-5, \quad h_{1}=h_{2}=0.1, d_{1}=d_{2}=0.09, m_{1}=m_{2}=0.09, L_{1}=L_{2}=4.5582\right)$

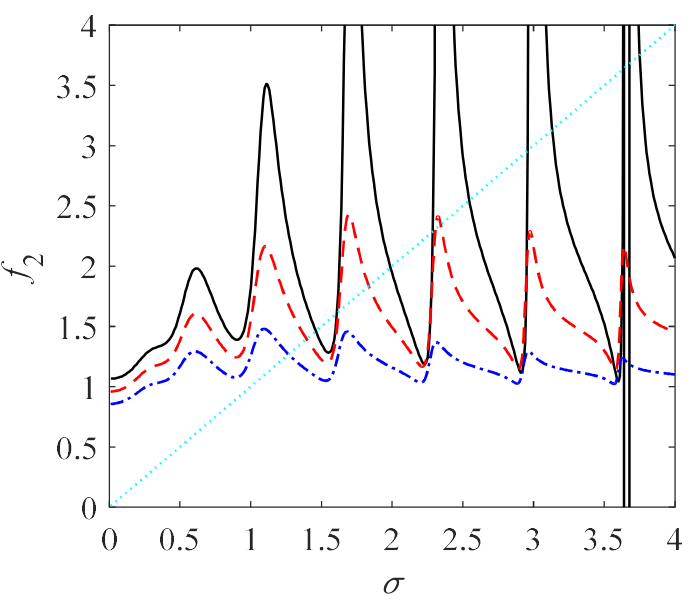

(a)

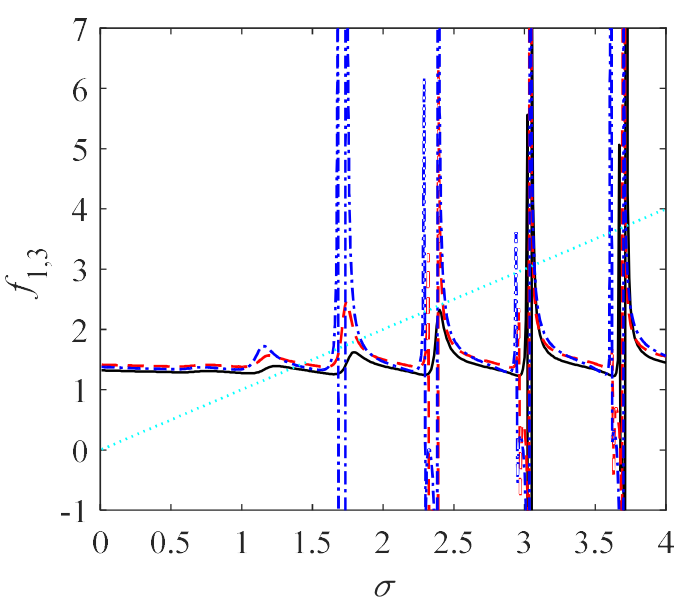

(b) 
Fig. 16. Natural frequencies of the floating elliptical cylinder. $(a)$ curves of function $f_{2},(b)$ curves of function $f_{1,3}$, solid lines are for $b / a=0.25$, dashed lines are for $b / a=0.5$, dash-dotted lines are for $b / a=0.75$. Dotted lines are for function $f$.

\section{Conclusions}

A hybrid method has been proposed to investigate the interaction of waves with a body floating on polynya confined between two semi-infinite ice sheets. The effectiveness and accuracy of the numerical scheme is demonstrated through two test cases, a submerged elliptical cylinder and a floating rectangle. Results are compared with those published and very good agreement is achieved. Extensive simulations are then carried out for an elliptical cylinder floating on polynya, from which the following conclusions can be drawn:

(1) The hybrid method is an effective approach for the wave/body/ice interaction problem, in which there are mixed free surface and ice sheet boundary conditions on a horizontal plane. It combines the strength of the methods in each corresponding domain. The method can be further extended to a much wider range of problems.

(2) The hydrodynamic force is highly oscillatory with the variation of the frequency. Its peak and trough are quite sensitive to the draught of the ice. Away from these extrema, the effect of the draught is small.

(3) As in the open water without the ice, the damping coefficient and excitation force can be related to the amplitude of the radiated wave at infinity. The oscillation of the former is closely linked to the oscillation of the latter.

(4) The natural frequency of the oscillating system, based on the usual definition, is obtained from the equation in which the inertial term is cancelled by the restoring force. This leads to multiple natural frequencies within a relatively small range. The wave damping can also be small at these frequencies due to its oscillatory behavior and very large motion can be excited by the incoming wave.

For a practical problem, as the frequency increases, the structural damping of the ice sheet may become important, which is not accounted for in the present work. As wavelength decreases, the thin plate model for the ice sheet may also need to be modified. Furthermore, the present work is limited to ice sheet of uniform properties. All these form part of the work for future, together with the extension of the method into the three dimensional problem.

\section{Acknowledgement}

This work is supported by Lloyd's Register Foundation through the joint centre involving University College London, Shanghai Jiaotong University and Harbin Engineering University, to 
which the authors are most grateful. Lloyd's Register Foundation helps to protect life and property by supporting engineering-related education, public engagement, and the application of research. This work is also supported by the National Natural Science Foundation of China (Grant No. 11472088 and 51709131)

\section{References}

Abramowitz, M., Stegun, I.A., 1965. Handbook of mathematical functions. Dover press, New York City.

Appolonov, E.M., Sazonov, K.E., Dobrodeev, A.A., Klementieva, N.Y., Kudrin, M.A., Maslich, E.A., Petinov, V.O., Shaposhnikov, V.M., 2013. Studies for development of technologies to make a wide channel in ice, The 22nd International Conference on Port and Ocean Engineering under Arctic Conditions, Espoo, Finland.

Bai, K.J., Yeung, R.W., 1974. Numerical solutions to free-surface flow problems, 10th Symp. Naval Hydrodyn., Cambridge, Mass., pp. 609-633.

Balmforth, N.J., Craster, R.V., 1999. Ocean waves and ice sheets. Journal of Fluid Mechanics 395, $89-124$.

Chung, H., Fox, C., 2002. Calculation of wave-ice interaction using the wiener-hopf technique. New Zealand J Math 31, 1-18.

Eatock Taylor, R., Zietsman, J., 1981. A comparison of localized finite element formulations for two-dimensional wave diffraction and radiation problems. International Journal for Numerical Methods in Engineering 17, 1355-1384.

Evans, D.V., Davies, T.V., 1968. Wave-ice interaction, Report 1313, Davidson Laboratory, Stevens Institute of Technology, New Jersey, .

Fox, C., Squire, V.A., 1990. Reflection and transmission characteristics at the edge of shore fast sea ice. Journal of Geophysical Research Oceans 95, 11629-11639.

Fox, C., Squire, V.A., 1994. On the oblique reflexion and transmission of ocean waves at shore fast sea ice. Philosophical Transactions of the Royal Society A347, 185-218.

Linton, C.M., Chung, H., 2003. Reflection and transmission at the ocean/sea-ice boundary. Wave Motion 38, 43-52.

Mei, C.C., Stiassnie, M., Yue, K.P., 2005. Theory and applications of ocean surface waves part 1: linear aspects. World Scientific Publishing Co. Pte. Ltd.

Meylan, M., Sturova, I., 2009. Time-dependent motion of a two-dimensional floating elastic plate. Journal of Fluids and Structures 25, 445-460.

Mohapatra, S.C., Ghoshal, R., Sahoo, T., 2013. Effect of compression on wave diffraction by a floating elastic plate. Journal of Fluids and Structures 36, 124-135. 
Newman, J.N., 1977. Marine hydrodynamics. MA: MIT Press, Cambridge.

Porter, R., Evans, D., 2007. Diffraction of flexural waves by finite straight cracks in an elastic sheet over water. Journal of Fluids and Structures 23, 309-327.

Ren, K., Wu, G.X., Thomas, G.A., 2016. Wave excited motion of a body floating on water confined between two semi-infinite ice sheets. Physics of Fluids 28, 20.

Robin, G.D.Q., 1963. Wave propagation through fields of pack ice. Philosophical Transactions of the Royal Society B255, 313-339.

Sahoo, T., Yip, T.L., Chwang, A.T., 2001. Scattering of surface waves by a semi-infinite floating elastic plate. Physics of Fluids 13, 3215-3222.

Squire, V.A., 2007. Of ocean waves and sea-ice revisited. Cold Regions Science \& Technology 49, $110-133$

Squire, V.A., 2011. Past, present and impendent hydroelastic challenges in the polar and subpolar seas. Philosophical Transactions of the Royal Society A369, 2813-2831.

Squire, V.A., Dugan, J.P., Wadhams, P., Rottier, P.J., Liu, A.K., 1995. Of ocean waves and sea ice. Annual Review of Fluid Mechanics 27, 115-168.

Squire, V.A., Robinson, W.H., Langhorne, P.J., Haskell, T.G., 1988. Vehicles and aircraft on floating ice. Nature 333, 159-161.

Sturova, I.V., 2013. Unsteady three-dimensional sources in deep water with an elastic cover and their applications. Journal of Fluid Mechanics 730, 392-418.

Sturova, I.V., 2015. Radiation of waves by a cylinder submerged in water with ice floe or polynya. Journal of Fluid Mechanics 784, 373-395.

Tkacheva, L.A., 2001. Hydroelastic behavior of a floating plate in waves. Journal of Applied Mechanics \& Technical Physics 42, 991-996.

Tkacheva, L.A., 2004. The diffraction of surface waves by a floating elastic plate at oblique incidence. Journal of Applied Mathematics \& Mechanics 68, 425-436.

Wehausen, J.V., Laitone, E.V., 1960. Surface waves, Handbuch des Physik. Springer, Berlin Verlag, pp. 446-778.

Williams, T.D., Porter, R., 2009. The effect of submergence on the scattering by the interface between two semi-infinite sheets. Journal of Fluids and Structures 25, 777-793.

Wu, G.X., 1991. Hydrodynamic forces on a submerged cylinder advancing in water waves of finite depth. Journal of Fluid Mechanics 224, 645-659.

Wu, G.X., Eatock Taylor, R., 1987. Hydrodynamic forces on submerged oscillating cylinders at forward speed. Proceedings of the Royal Society of London. A. Mathematical and Physical Sciences 414, 149-170. 
Wu, G.X., Eatock Taylor, R., 2003. The coupled finite element and boundary element analysis of nonlinear interactions between waves and bodies. Ocean Engineering 30, 387-400.

Yeung, R.W., 1975. A Hybrid Integral-Equation Method for Time-Harmonic Free-Surface Flows, 1st Int. Conf. Numer. Ship Hydrodyn., Gaithersburg, Maryland, pp. 581-608.

Yeung, R.W., Bouger, Y.C., 1979. A hybrid integral-equation method for steady two-dimensional ship waves. International Journal for Numerical Methods in Engineering 14, 317-336. 